\title{
Planar nonlinear metasurface optics and their applications
}

\author{
Tianye Huang ${ }^{\dagger}$, Xiang Zhao ${ }^{\dagger}$, Shuwen Zeng ${ }^{*}$, Aurelian Crunteanu, Perry Ping Shum*, and \\ Nanfang $\mathrm{Yu}^{*}$
}

\section{Abstract}

Metasurfaces are artificial two-dimensional (2D) planar surfaces that consist of subwavelength "meta-atoms" (i.e., metallic or dielectric nanostructures). They have been known for the capability to achieve better and more efficient light control in comparison to their traditional optical counterparts. Abrupt and sharp changes in the electromagnetic properties can be induced by the metasurfaces rather than the conventional gradual accumulation that requires more propagation distances. Based on this feature, planar optical components like mirrors, lenses, waveplates, isolators and even holograms with ultrasmall thicknesses have been developed. Most of the current metasurface studies focused on tailoring the linear optical effects for applications such as cloaking, lens imaging and three-dimensional holography. Recently, the uses of metasurfaces to enhance nonlinear optical effects have attracted significant attention from the research community. Benefiting from the resulted efficient nonlinear optical processes, the fabrication of integrated all-optical nano-devices with peculiar functionalities including broadband frequency conversions and ultrafast optical switching will become achievable. Plasmonic excitation is one of the most effective approaches to increase the nonlinear optical responses due to its induced strong local electromagnetic field enhancement. For instance, continuous phase control on the effective nonlinear polarizability of plasmonic metasurfaces has been demonstrated through spin-rotation light coupling. The phase of the nonlinear polarization can be continuously tuned by spatially changing the "meta-atoms" orientations during second and third harmonic generation (SHG/THG) processes, while the nonlinear metasurfaces also exhibit homogeneous linear properties. In addition, an ultrahigh secondorder nonlinear susceptibility up to $10^{4} \mathrm{pm} / \mathrm{V}$ has recently been reported by coupling the plasmonic modes of patterned metallic arrays with intersubband transition of multi-quantum-well layered substrate. In order to develop ultra-planar nonlinear plasmonic metasurfaces, two-dimensional materials such as graphene and transition metal dichalcogenides (TMDCs) have been extensively studied based on their unique nonlinear optical properties. The third-order nonlinear coefficient of graphene is 5 times as that of gold substrate, while TMDC materials also exhibit a strong second-order magnetic susceptibility. In this review, we first focus on the main principles of planar nonlinear plasmonics based on metasurfaces and 2D nonlinear materials. The advantages and challenges for incorporating 2D nonlinear materials into metasurfaces have been discussed, 
followed by their potential applications including orbital angular momentum (OAM) manipulating, and quantum optics.

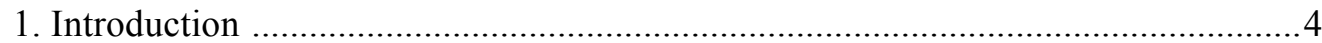

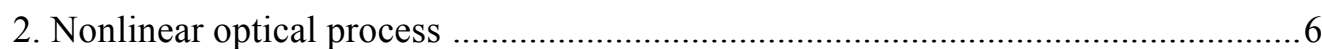

2.1 Nonlinear response enhancement by plasmon ......................................... 8

2.2 Optical properties of graphene and other 2D materials ............................. 16

2.2a Nonlinear optical properties of graphene ............................................ 16

2.2b Nonlinear optical properties of TMDCs .......................................... 16

2.2c Nonlinear optical properties of ENZ materials ................................... 18

2.3 Application of nonlinear graphene and other 2D materials ........................... 18

2.3a Graphene with plasmon resonator.............................................20

2.3b Graphene by chemical doping or electric doping ................................21

$2.3 \mathrm{c}$ Patterned graphene .........................................................................22

2.3d Structure with 2D TMDCs materials ............................................24

2.3e Structure with ENZ dielectric materials............................................26

3. Nonlinear metasurfaces......................................................................... 28

3.1 Arrays of non-centrosymmetric metal nanoparticles ..............................28

3.2 Arrays of semiconductor materials ...................................................... 30

3.3 Fano resonance based metasurfaces........................................................ 32

3.4 Plasmonic metasurfaces coupled to intersubband transitions .......................33

3.5 Nonlinear waveguides coated with phase gradient metasurfaces .....................36

4. Incorporate graphene and other 2D materials into metasurfaces .......................37



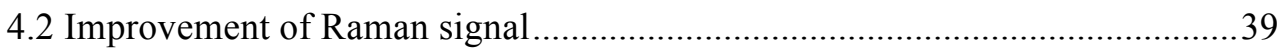


4.3 Enhancement of FWM and phase conjugation . .41

4.4 Efficient generation of high harmonics. .43

5. Summary and future outlook .44 


\section{Planar nonlinear metasurface optics and their applications}

\section{Introduction}

With the rapid development of micro-nano processing methods, researchers currently can artificially control the flow of light through a set of designs with various types of metal and dielectric nanostructures [1-5]. Metamaterials are artificial composites with unique optical properties generated from subwavelength metal-dielectric resonance elements that are called meta-atoms. These artificially engineered structures provide significant potential for controlling the flow of electromagnetic energy especially in the engineering space area [6], by customizing the composition of metamaterials and adjusting the shape and size of structural elements. Based on these methods, multiple novel functions such as cloaking, super lens and higher-order topological insulators are recently achieved. And they are not observed in natural materials. However, due to the limitation of planar manufacturing technology, current researches of optical metamaterials mainly focus on three-dimensional (3D) transformation optics of bulk material characteristics [7,8]. The emergence of metasurfaces breaks the requirement on propagation effects [9-13] and make the use of them to boost up various application fields such as negative refraction [14], perfect lenses [15], optical hiding [16], and Nano-resolution imaging [7]. Metasurfaces are defined as two-dimensional planar materials consisting of arrays of scatters or thin films that enables control of light in a plane. The separation and geometric parameters of the optical scatters are engineered to manipulate the interaction with light, e.g. arrays of metal or dielectric nanoparticles [17, 18]. In this way, one can obtain abrupt and controllable optical characteristics. More importantly, optical thin films made of lossy material can also form metasurfaces with different properties $[19,20]$ since the reflected light or transmitted light at the interface of lossy material also experiences significant interfacial phase change. Metasurfaces are initially described for employment in mid-infrared wavelengths [10] due to the more facile synthesis of sub-micrometer arrays, and now extend to the near-infrared wavelength region in terms of nanoarrays [21]. It is worth noting that the 2D metasurfaces show superior compatibility with on-chip nanophotonic fabrication techniques, it thus plays a vital role in the development of integrated planar optical devices as new generation of antennas, microscopes, photodetectors and sensors [13]. 
Another intriguing part for the metasurfaces is that they also offer strong nonlinear response benefiting from the strong resonance enhancement inside. This property paves a way to achieve all-optical signal processing in one circuit [22]. Optical nonlinearity is defined as the change in the optical properties of the medium when the electric or magnetic fields are introduced, or the strength of the field is enhanced [23]. It is known that the strong interaction between photons and conventional continuous thin film materials is difficult to excite by the light source, leading to weak optical nonlinearity. However, this limitation can be improved by field enhancement originated from the surrounding environment. Surface plasmons are electromagnetic modes generated by the interaction between light and surface charges, such as free electrons in metals. Due to the interaction of light and matter, the momentum of surface plasmons is larger than that of light of the same frequency in vacuum, which limits the corresponding electromagnetic field to the surface. Therefore, the electromagnetic field can be significantly enhanced in this condition [24-26]. The optical nonlinearity can be significantly improved by the strong field enhancement in surface wave at the metal-dielectric interface. For the 2D metasurfaces, metallic nanoparticles are usually designed in the structure to produce local surface plasmons. The optical nonlinearity of the metasurface structures can be enhanced by tuning the local surface plasmon interactions between the nanoparticles.

In order to develop the highly-desired integrated devices based on ultra-planar nonlinear plasmonic metasurfaces, atomically thin graphene is considered to be a much more promising candidate compared to noble metals. Graphene is an atomic thin carbon layer arranged in a two-dimensional honeycomb lattice. It has much stronger inherent nonlinear effects than most of the bulk semiconductor materials [27]. High nonlinear refractive index, high THG coefficient and four wave mixing (FWM) efficiency are observed in graphene [27-30]. Moreover, the monolayer of graphene can further enhance the nonlinear optical response by better confining the plasmon wave to the graphene surface [31,32]. Both theoretical and experimental studies [33-39] have shown that graphene can confine plasmon with mode volume millions of times smaller than free space. The electrical conductivity of graphene can be tuned by different approaches such as chemical doping and electrical doping. Gate injected charge carriers in the optoelectronic devices can be applied to effectively reduce the optical response time. In addition, much low-energy tunable plasmons are observed inside patterned graphene including expanded graphene (EG) [40-44], graphene bands [45-47], disks [48, 49], rings [50], disk stacks [50, 51], and voids [52]. By engineering the doped graphene and patterned graphene nanostructures provide a unique and practical opportunity to achieve highly efficient nonlinear metasurfaces. 
In this paper, we review the recent progress in nonlinear metasurfaces and their applications ranging from photonics devices (optical switch and modulators), high order signal generation, to chemical and biosensing. In Section 2, we firstly brief introduction on principles of optical nonlinearity, method for nonlinear enhancement through structure design, and theory on nonlinear metasurfaces. In Section 3, we discuss the optical properties of graphene, including supporting and confining plasmons and the plasmon-enhanced light absorption for non-linear generation. We also review the intrinsic nonlinearity of graphene. In Section 4, the latest trend on the applications based on graphene metasurfaces and novel metastructure incorporated with graphene. Finally, Section 5 summarizes the advantages and future challenges of the patterned metasurfaces.

\section{Nonlinear optical process}

The polarization of optical medium mainly describes the electromagnetic response in applied electric field at optical frequency. In the electric dipole approximation, the nonlinear polarization is defined as the power expansion of the external electric field in the form of a Taylor expansion:

$$
P(\mathrm{E})=\varepsilon_{0}\left[\chi^{(1)} E+\chi^{(2)} E^{2}+\chi^{(3)} E^{3}+\cdots \chi^{(N)} E^{N}\right]
$$

where $E$ represents the strength of electric field, $\varepsilon_{0}$ is the permittivity of free space, and $\chi^{(\mathrm{n})}$ is the $\mathrm{n}^{\text {th }}$ order susceptibility of optical medium. The first term in equation (1) plays an important part in linear optics, which related with conventional optical phenomena such as reflection, refraction, absorption and scattering. But under the condition with strong fields, the higher-order terms in this expansion form nonlinear optical response. It is worth noting that $\chi^{(2)}$ of the material with central symmetry is zero, and therefore second-order nonlinear optical effects need to be acquired from higher order effects, including magnetic dipoles and electric quadrupoles. In this context, $\chi^{(3)}$ is the dominant nonlinear interaction described for plasmonic materials. However, due to the lack of inverted symmetry at the material interface itself, the deviation from perfect lattice periodically breaks inverted symmetry in the strain system [53], or a sufficiently strong electrostatic field induces asymmetry. In this condition, $\chi^{(2)}$ is induced at the material surface.

This second order nonlinear polarization leads to nonlinear wave mixing as shown in Fig. 1. Supposing the incident field consists of two different frequencies, $\omega_{1}$ and $\omega_{2}$, a series of important nonlinear optical processes at the doubled frequencies, such as second harmonic 
generation ( $\mathrm{SHG}$ ) at $2 \omega_{1}$, sum-frequency generation (SFG) at $\omega_{1}+\omega_{2}$, and differencefrequency generation (DFG) at $\omega_{1}-\omega_{2}$ are depicted. Based on the above phenomenon, thirdorder nonlinear processes are highly desired over the second-order ones. THG occurs when the frequency of a photon $3 \omega$ generated from three incident photons at frequency $\omega$, while four-wave mixing (FWM) is induced with two light waves at one specific frequency encountered in a nonlinear material. In this case, two signals with new frequency are generated and interact with each other. As we all know that the most primary third order nonlinear optical process studied in general nonlinear optics is the Kerr effect, in which the third-order response contains oscillations also at the fundamental frequencies, leading to the nonlinear refractive index given by:

$$
n=n_{0}+n_{2} I
$$

where $n_{0}$ is the linear refractive index, $\mathrm{n}_{2}$ is the nonlinear index coefficient, and $I$ is the optical intensity, $n$ is thus the effective refractive index of the medium. The corresponding dielectric constant $\varepsilon$ is expressed by:

$$
\varepsilon=\varepsilon_{0}\left[\chi^{(1)}+3 \chi^{(3)}|E|^{2}\right]
$$

where $\varepsilon_{0}$ is the vacuum permittivity.

(a)



(c)

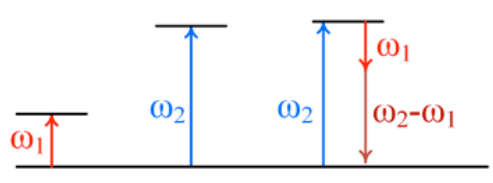

(b)


second order nonlinear process is (a) SHG, (b) SFG, and (c) DFG, respectively.

In bulk nonlinear media, the required condition for the parametric nonlinear process is the phase matching, which ensures the generated optical signals constructively build up and then the optical power will be transmitted continuously from the pump to the signal. Normally, the nonlinear parametric effects described above are difficult to be observed simultaneously due 
to their different phase matching conditions respectively. On the nanoscale dimension, however, phase matching is relaxed. And in this condition, optical fields are strongly confined within or surround the nanostructures, nonlinear interactions can therefore be realized with an ultra-small area down to $10 \mathrm{~nm}$ to $100 \mathrm{~nm}$ and can be scattered in all directions to produce more frequencies.

\subsection{Nonlinear response enhancement by plasmon}

Optical nonlinearities are inherently weak and originate from photon-photon interactions supported in the material. They are affected by electromagnetic fields and thus can be enhanced in the physical environment that provides the field enhancement. Plasmonic is an electromagnetic oscillation caused by the charge density fluctuation on a metal-dielectric interface when incident light interacts with metal nanostructures. Here, the charge density wave is closely related to electromagnetic wave which propagates parallel to the plane at the interface of two media and called surface plasma wave. The electric field vector reaches its maximum value at the interface where the interaction between light and matter can be significantly improved. And this field intensity attenuates exponentially with the propagation. The propagation constant of the surface plasma wave could be expressed as:

$$
\beta=k_{0} \sqrt{\frac{\varepsilon_{m} n_{s}{ }^{2}}{\varepsilon_{m}+n_{s}{ }^{2}}}
$$

where $k_{0}$ is the wave vector in free space, $\varepsilon_{\mathrm{m}}=\varepsilon_{\mathrm{mr}}+i \varepsilon_{\mathrm{mi}}$ denotes the dielectric constant of the metal, $\varepsilon_{\mathrm{mr}}$ and $\varepsilon_{\mathrm{mi}}$ represent the real part and the imaginary part of the dielectric constant respectively. And $n_{\mathrm{s}}$ is the refractive index of the dielectric layer on top of the metal surface. The equation (3) imply that the surface plasma wave could be excited when $\varepsilon_{\mathrm{mr}}<-n_{\mathrm{s}}{ }^{2}$. The surface plasma wave was first described by Wood in 1902, where he observed an abnormal diffraction phenomenon on the metallic diffraction grating [54]. Since the phase velocity of surface plasma waves on a metal surface is smaller than the velocity of the incoming light, the light incident on the surface cannot excite these waves. Thus, a method for excitation of nonradiating surface plasma waves on smooth surfaces was shown by otto and kretschmann in 1968. By placing a prism close to a sliver interface, surface plasma waves were excited by evanescent waves in total reflection [55]. In addition to prism coupling and diffraction gratings, optical waveguide structures could also realize the surface plasma waves excitation, as shown in Fig. 2. Dated back from 1961, the nonlinear optical process was first experimentally demonstrated in terms of second harmonic generation from a quartz crystal with the pump through a ruby laser beam. The coupling of SHG to surface plasmon mode was 
reported by H. j. Simon et al in 1974 theoretically. The phenomenon of reflected secondharmonic generating from media with inversion symmetry was firstly reported to be observed in silver (Ag) thin films. After than this phenomenon had been systematically studied both experimentally and theoretically. It was found that the resonantly enhanced SHG was directly linked and had strong relation to the surface plasmon excitation [56].

a)

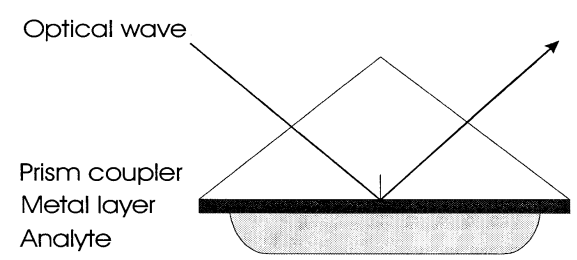

b)
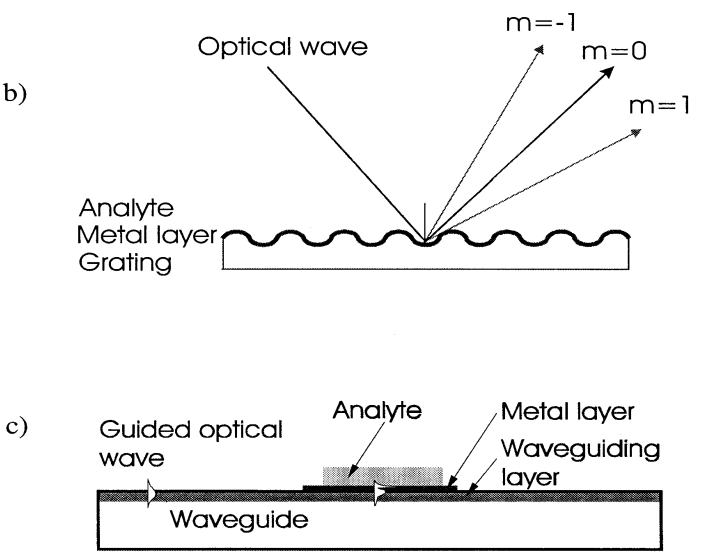

Fig. 2. Different structure for exciting SPP. (a) Prism coupling. (b) Grating coupling. (c)

$$
\text { Waveguide structure. }
$$

The resonance associated with free electron motion supported by nanoparticles is called local surface plasma resonance (LSPR). The intrinsic frequency of the local plasmon is obtained:

$$
\frac{\partial^{2} \rho}{\partial \mathrm{t}^{2}}+\omega_{p}^{2}\left(\rho-\rho_{0}\right)=0
$$

where $\rho(t)$ is the electron density of nanoparticle and $\rho_{0}$ is the average density. When the frequency of the incident electromagnetic field equals that frequency, the LSPR of the nanoparticle is excited, resulting in a rapid enhancement of the plasmon wave.

LSPR leads to the enhancement and localization of electromagnetic fields near metal nanoparticles and also the enhanced scattering cross section. In addition, LSPRs may generate enhanced nonlinear light-matter interactions that can significantly improve the nonlinear 
processes. Assuming that LSPR occurs at the surface of metal nanosphere, the size of the nanosphere is smaller than the excitation wavelength. When one determines the field near the surface, the dielectric constant inside the metal nanosphere is represented by $\varepsilon_{\mathrm{i}}\left(\varepsilon_{\mathrm{i}}\right.$ is independent with the size of the nanosphere), and it is assumed that the nanosphere is embedded in a medium with a dielectric constant $\varepsilon_{0}$. The electric field of the incident electromagnetic wave is set to $\mathbf{E}_{\mathrm{o}}$, which is defined as a vector pointing in the direction of the z-axis. The Maxwell equations can be used with the approximation of the Laplace equations to determine the electric field inside and outside the spherical nanoparticle. The result field $\mathbf{E}_{\text {out }}$ outside the nanosphere can be written as [57]:

$$
\mathbf{E}_{\text {out }}=E_{o} \mathbf{z}-\alpha E_{o}\left[\frac{\mathbf{z}}{r^{3}}-\frac{3 z}{r^{5}}(z \mathbf{z}+x \mathbf{x}+y \mathbf{y})\right]
$$

where $x, y, z$ are the usual Cartesian coordinates, $\mathrm{r}$ is the radial distance, $\mathbf{x}, \mathbf{y}, \mathbf{z}$, represent the Cartesian unit vectors, $E_{\mathrm{o}}$ is the magnitude of $\mathbf{E}_{\mathrm{o}}$, and $\alpha=g a^{3}$ is the polarizability of nanosphere, where $a$ is the radius of nanosphere, $g=\frac{\varepsilon_{i}-\varepsilon_{o}}{\varepsilon_{i}+2 \varepsilon_{o}}$, the second term is the induced dipole produced by the polarization of the electron density of nanosphere. Polarizability is a number that is in the order of the spherical nanoparticle with the limit of zero frequency, in which case $\varepsilon_{\mathrm{i}}$ equals to one. However, when the real part of $\varepsilon_{\mathrm{i}}$ equals to $-2 \varepsilon_{\mathrm{o}}$ and the imaginary part of $\varepsilon_{\mathrm{i}}$ is smaller, $\alpha$ will become larger. In this way, the induced electric field will be thus enhanced.

For a single plasmonic particle, there are only a few studies devoted to analyzing them for their intrinsic nonlinear properties [58-60], due to the weak second-order nonlinear interaction. In small particles, whose sizes are smaller than the wavelength of incident light, the secondorder processes also disappear due to central symmetry [61]. However, off-center symmetrical structure will still produce SH intensity. In this case, the SH response is mainly determined by the electric dipole contribution that is resulted from the shape centrosymmetry breaking [62, 63].

For metal nanoarrays, nonlinear emission is described by assuming that there is a local nonlinear susceptibility tensor on the metallic surface based on the nonlinear scattering theory. The nonlinear emission can be determined by Lorentz's reciprocity theorem [64]:

$$
E_{n l}(2 \omega) \propto \iint \chi_{n n n} E_{n}^{2}(\omega) E_{n}(2 \omega) d S
$$

In this equation, $E_{\mathrm{nl}}(2 \omega)$ represents the nonlinear emission, $\chi_{\mathrm{nnn}}$ is the local nonlinear susceptibility, and $E_{\mathrm{n}}(\omega)$ and $E_{\mathrm{n}}(2 \omega)$ are the linear fields of the fundamental mode and the 
second harmonic frequency mode to the nanostructure surface. This process is shown in Fig. 3. And the value of the overlap integral is dependent on the contribution of the local field and related signals. Destructive interference would occur and lead to low nonlinear scattering. On the contrary, constructive interference between the nonlinear polarization mode and the corresponding harmonics would generate a more significant far-field nonlinear emission. Thus, the microscopic linear polarization is closely related to far-field emission [6].

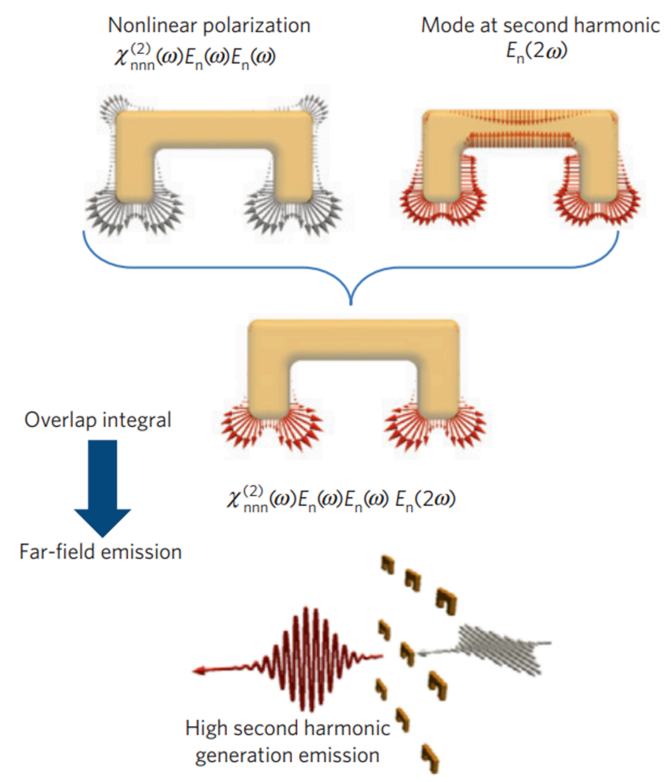

Fig. 3. Nonlinear scattering theory. The overlap integral of the microscopic nonlinear polarization, calculated from the linear fields at the fundamental frequency $\omega$ (for normal incidence), and the mode at the second harmonic yields the far-field nonlinear emission [6].

Due to the ability of confining the incident electromagnetic radiation into the near-field area, plasmonic systems can significantly enhance weak nonlinear processes, providing an opportunity to control light constraints at the nanoscale. In addition to nonlinear effects with ultra-fast response times, the plasmon excitation parameters are extremely sensitive to the dielectric properties of the metal and the surrounding dielectric. This characteristic can be employed to modify the resonance property and signal propagation by inducing the nonlinear change of the dielectric properties of the material. Furthermore, benefiting from the nanostructures, phase matching is not an essential condition with their dimensions much smaller than the wavelength. In fact, the harmonic generation in plasmonic nanostructures is described more as a nonlinear scattering phenomenon rather than a phase matching process. The wave incident on the nonlinear material at the fundamental frequency $\omega$ rises to the oscillation source polarization at the SH frequency $2 \omega$. When the source is a single atom or 
molecule, SH emission occurs in all directions, as shown in Fig. 4 (a). But for thicker samples, the oscillation of the wave during propagation becomes critical and its rate is proportional to the refractive index. The source oscillation follows the fundamental field, but occurs twice as fast. Moreover, due to the higher frequency, the refractive index of the SH wavelet is different, and the SH wavelet at different positions will lose the phase relationship after the distance of the coherent length, as shown in Fig. 4(b). The coherent length of the forward SH generation process is generally around $10 \mu \mathrm{m}$, while in the backward process it is only about $100 \mathrm{~nm}$, leading to much weaker nonlinear interaction [65]. To effectively enhance weak nonlinear processes, many plasmons excitation methods have been designed and reported as summarized in Fig. 5, most of which are based on surface plasmons excited by metal thin films, local surface plasmon excited by metal nanoparticle, Fano resonance induced by the coupling between dark and bright mode, the combination of the nonlinear active materials and passive materials, nonlinear 2D materials, and nonlinear beam shaping by a gradual variation of the geometrical structure of the meta-atoms along the metasurfaces.
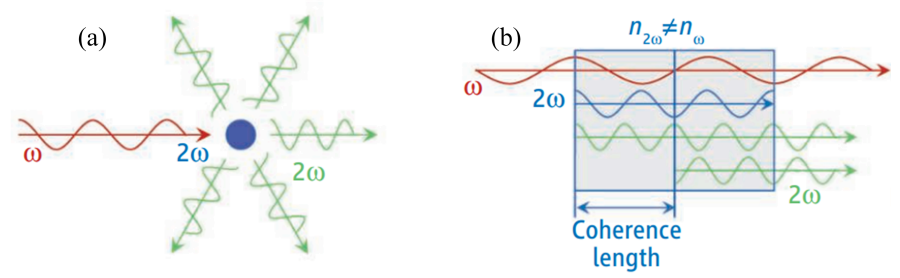

Fig. 4. Phase matching for SH generation. The oscillations at fundamental wave $\omega$ is shown by red lines, SH source polarization at the SH frequency $2 \omega$ is shown by blue lines, and green lines indicate $\mathrm{SH}$ wavelets. (a) SH source from single atom or molecule emits in all directions. (b) For thick samples, the phase relation between the SH wavelets is lost after a coherence length, because the source and wavelets oscillate at different rates [65]. 


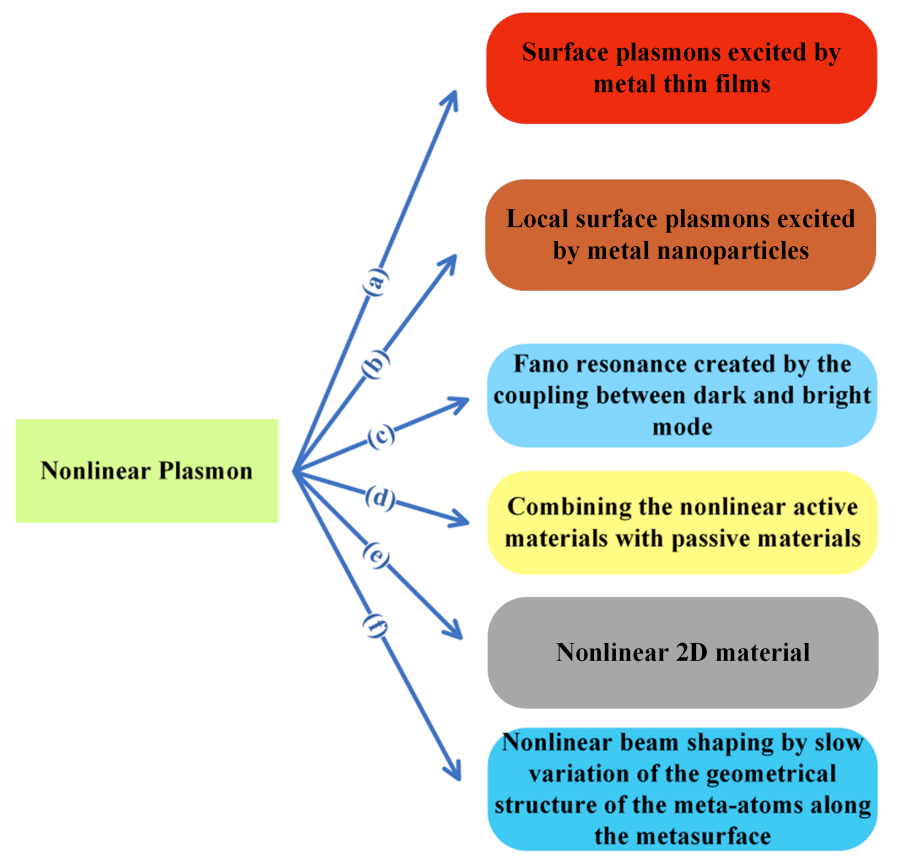

Fig. 5. Various plasmons excited methods to enhance nonlinear processes. (a) Surface plasmons excited by metal thin films, (b) Local surface plasmon excited by metal nanoparticle, (c) Fano resonance induced by the coupling between dark and bright mode, (d) Combining the nonlinear active materials with passive materials, (e) Nonlinear 2D materials. (f) Nonlinear beam shaping by slow variation of the geometrical structure of the meta-atoms along the metasurface.

The second-order and third-order processes on various types of surfaces have been extensively studied, in which the geometric shape on the substrate is designed to achieve a coherent and directional response [66]. J. Renger employed a nanostructured gold surface to convert the evanescent FWM waves into propagating radiation, as shown in Fig. 6(a). Two laser beams with frequencies $\omega_{1}$ and $\omega_{2}$ are incident to the gold surface, respectively [67]. Efficiency value of FWM changing with excitation angles is plotted in Fig. 6(b). The choice of excitation angle not only defines the direction of FWM radiation but also strongly influences the efficiency of FWM. The metallic nanostructure also enhanced the in-and outcoupling of such radiation, thereby increasing the FWM efficiency significantly. In general, the signal of the second-order nonlinear optical process in the single particle is very weak. Thus, in order to obtain a strong harmonic response of single nanoparticle, the experiments where the second harmonic signal from a single metallic nanoparticle dispersed in a homogeneous medium was achieved by J. Butet's group for the first time, as shown in Fig. 6(c) [61]. The observation of interference with the SHG between selected dipoles and octupoles generated from spherical gold nanocrystals was also demonstrated in Fig. 6(d). It 
was the earliest report about octupole response of particles in the harmonic field component which was in the parallel direction of the scattering plane [68]. Fano resonances (FRs), which are generated by the coupling between the dark mode and the bright mode in the resonance system, can enable the ability to tailor the fundamental near-field distribution and consequently control the nonlinear surface polarization, it has been demonstrated that FRs are able to enhance optical nonlinear effects. S. Liu et al. proposed a plasmonic nonamer in a cross structure surrounded by eight gold nanorods together to sustain polarizationindependent FRs in the NIR region with higher-order plasmon resonances induced for the excitation at the visible, as shown in Fig. 6(e). Multiple Fano resonances result from the plasmon coupling of the near-field interaction between the nano-crossing layer and the two outer ring layers. The radiation losses of nonamers are simultaneously suppressed at all spectral positions in the near infrared region attributed to the simultaneous excitation of multiple Fano resonances. The high order resonant mode generated by the multipole Fano resonances in the cross structure of the nanorods could be coupled with the nonlinear signal, resulting in the enhanced scattering of $\mathrm{SH}$ emission. The asymmetric angular intensity distribution shown in Fig. 6(f) is inconsistent with the dipole radiation, confirming the contribution of higher multipoles to SH emission intensity [69]. Combining the nonlinear active materials with passive materials can significantly enhance the nonlinear process. Utilizing the plasmon-mediated field constraints, the traditional nonlinear optical materials can be coupled in the near-field amplification area of the plasmonic nano-structures. This would significantly improve the pump intensity and also enhance the nonlinear conversion efficiency of harmonic generation and multi-wave mixing from active nanoparticles. $\mathrm{H}$. Aouani et al. used individual semiconductor indium tin oxide (ITO) nanoparticles (NPs) to enhance THG as well when coupled with a gold nanodimer. As shown in Fig. 6(g), the gold nanodimer served as a received antenna [17]. It confined the incident far-field radiation to a near field localized between their gaps. The ITO NPs that located at the gap further upconverted incident photons from frequency of $\omega$ to the one with frequency of $3 \omega$. Fig. 6(h) show the three-photon up-conversion enhancement where it indicated that the single ITO nanoparticle conjugated with a gap nanorod plasmonic dimer led to a dramatic enhancement on the originally weak THG efficiency. [70]. 



(g)


(d)
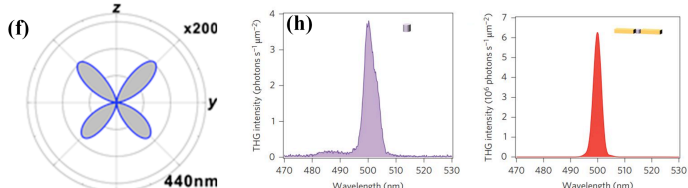

Fig. 6. Experiments and structure of excitation of plasmons to enhance nonlinear processes. (a) A nanostructured gold surface to enhance FWM, two incident beams with frequencies $\omega_{1}$ and $\omega_{2}$ give rise to reflected beams with frequencies $\omega_{\text {Fwm } 1}$ and $\omega_{\text {Fwm } 2 \text {, }}$ respectively. (J. Renger). Reprinted with permission from [67]. Copyrights (2010) American Physical Society. (b) Efficiency of FWM as a function of excitation angles $\theta_{1}$ and $\theta_{2}$. The solid line indicates the boundary between propagating and evanescent FWM waves. [67]. (c) The SH intensity for gold nanoparticles embedded in gelatin with low (Zone 1) and high (Zone 2) intensities (J. Butet et al.) [61]. (d) Vertically polarized SH intensity of Zone 1 and Zone 2 [61]. (e) Schematic of a gold nonamer composed of a cross structure surrounded by eight gold nanorods (S. Liu et al.)[69]. (f) The angular plot of the second-harmonic far-field emission intensity perpendicular to the sample surface [69]. (g) Schematic of third-harmonic radiation from a single ITO nanoparticle with a plasmonic nanorod dimer (H. Aouani et al.). Reprinted with permission from [70]. Copyrights (2014) Springer Nature. (h) The three-photon upconversion enhancement with and without the single ITO nanoparticle is decorated with a gap nanorod plasmonic dimer [70]. 


\subsection{Nonlinear optical properties of graphene and other $2 D$ materials}

\section{2a Nonlinear optical properties of graphene}

As a centrosymmetric material, second-order nonlinear interactions are known to be forbidden within graphene. However, the second-order nonlinear response of graphene can be described by third order conductivity influenced from the interface effects [71], nonuniformity of optical field [72], or the presence of dc currents [73]. In the case of ignoring electron-electron and electron-phonon scattering processes with thermal effects, the third order conductivity for this effect is expressed by [74]:

$$
\sigma_{s}^{(3)}(\omega)=\frac{i \sigma_{0}\left(\hbar v_{F} e\right)^{2}}{48 \pi(\hbar \omega)^{4}} T\left(\frac{\hbar \omega}{2 \varepsilon_{F}}\right)
$$

where $\omega$ is the angular frequency, e indicates the electron charge, $\hbar$ is the reduced Planck's constant.

To study the third-order nonlinearity of graphene, the extraction of effective bulk susceptibilities is particularly important. This nonlinear susceptibility is useful in experimental investigations of graphene's nonlinear optical phenomena, as it relies heavily on measurement methods, the frequency of light, and sample preparation. The effective bulk susceptibility is given by:

$$
\chi_{b}^{(3)}=\frac{i}{\varepsilon_{0} \Omega_{t} h_{e f f}} \sigma_{s}^{(3)}
$$

where $h_{\mathrm{eff}}$ is the effective thickness of graphene, $\varepsilon_{0}$ is the permittivity of free space and $\Omega_{\mathrm{t}}=$ $3 \omega_{0}$ is the frequency at the third harmonic with $\omega_{0}$ being the fundamental frequency.

\section{2b Nonlinear optical properties of TMDCs}

Due to the confinement of electron motion and the lack of interlaminar perturbation, the $2 \mathrm{D}$ TMDCs as another novel 2D nanomaterials are considered a promising material in the emerging applications like nano-electronics and nano-optoelectronic high-performance devices [75-77]. Different from graphene, TMDCs monolayer belongs to the $\mathrm{D}_{3 \mathrm{~h}}$ point group [78], which means that they are non-centrosymmetric nanomaterials and SHG is the lowestorder nonlinear optical process. Therefore, the nonlinear susceptibility here is only an independent, nonvanishing component, which means:

$$
\chi_{b, 0}^{(2)}=\chi_{b, x x x}^{(2)}=-\chi_{b, x x y}^{(2)}=-\chi_{b, y x y}^{(2)}=-\chi_{b, y y x}^{(2)}
$$


where $x$ is the armchair direction of the single TMDC layer and $y$ is the orthogonal zigzag direction. The value of second-order susceptibility for $\mathrm{MoS}_{2}, \mathrm{WS}_{2}$ and $\mathrm{WSe}_{2}$ are $132 \mathrm{pm} / \mathrm{V}$ $1140 \mathrm{pm} / \mathrm{V}$ and $67 \mathrm{pm} / \mathrm{V}$, respectively, which show strong bulk second-order susceptibility [79]. Recently, black phosphorus as another promising 2D nanomaterials of which the structures are similar with those of TMDCs has attracted great attention for their enhancement function of nonlinear optical response [80]. Black phosphorus is a high carrier mobility and direct energy bandgap semiconductor with a bandgap of $2 \mathrm{eV}$ for single layer and a bulk-state bandgap of $0.3 \mathrm{eV}$ [81]. Due to their thickness-dependent bandgap and anomalous anisotropy, black phosphorus also shows nonlinear saturable absorption properties [82].

By applying Lorentz reciprocity, it is possible to accurately calculate the dipoles, quadrupoles, and higher-order contributions to the nonlinearity of nanostructures [83]. It means that with the assumption of an entirely weak nonlinear signal, the effective nonlinear response of the metasurface is linearly related to the spatial overlap integral between the fields excited in the structure through the probing of the pump frequency and SH frequency waves with a specific polarization combination, that is averaged over unit cell volume $V$ and weighted by local nonlinear susceptibility tensor [84]:

$$
\chi_{e f f, l m n}^{(2)}=\frac{1}{V} \sum_{i j k} \int_{V} \chi_{i j k}^{(2)}(\mathbf{r}) \frac{E_{i(l)}^{2 \omega}(\mathbf{r}) E_{j(m)}^{\omega}(\mathbf{r}) E_{k(n)}^{\omega}(\mathbf{r})}{E_{i n c, l}^{2 \omega} E_{i n c, m}^{\omega} E_{i n c, n}^{\omega}} d^{3} \mathbf{r}
$$

where $E_{i(l)}^{\omega}$ represents the $i$-polarized component of the electric field on the metasurface excited by $l$-polarized incident probing wave $E_{i n c, l}^{\omega}$, the average of unit cell volume will automatically eliminate the effect of dipole on SHG.

It is important that this equation is only valid under quasi-static conditions. For unit cells of the metasurfaces that are much smaller than the excitation wavelength at SH frequency, the volume average is invalid if this condition is not satisfied. In addition, to maximize the second harmonic signal, the metasurface structure needs to generate strong field enhancement at both $\omega$ and $2 \omega$. In this case, the structure should radiate the nonlinearity generated fields at the SH frequency effectively while maintaining a strong magnetic field at the pump frequency. For metal structures, nonlinearity only generates at the surface because the electric field decays rapidly from the excitation interface. The volume integral is reduced to the surface overlap integral over the metal boundary, the nanostructure with broken central symmetry can exhibit spatially asymmetric field overlap, resulting in a strong second-order nonlinear response. 


\section{2c Nonlinear optical properties of ENZ materials}

There is a special synergy between the near zero parameter structure and the nonlinear optical process. The near zero parameter structure mainly improves the phase matching and the high field intensity in order to enhance the nonlinear response of the material. The phase matching here is also able to reduce the destructive interference of the field caused by the nonlinear polarization source in general. However, in structures where the parameters are close to zero where photons with zero momentum, any combination of photon directions satisfies the momentum conservation principle, thus nonlinearly generated waves can be coherently established in the forward and backward directions. In this case, regardless of the directionality, the nonlinear process of the zero-refractive-index material is effective, and the need to balance the momentum of the waves involved in the nonlinear interaction is eliminated. As a result, nonlinear processes can only be observed at high field intensities, due to the competition of the excited electric field with the restoring force of the atomic field. Electric field strength is usually improved through confining it in a relatively small spatial region in the traditional devices, whereas nanostructures with epsilon near zero (ENZ) parameters could simultaneously generate strong field strength enhancement over large regions and provide phase matching [85].

\subsection{Applications of nonlinear graphene and other $2 D$ materials}

Due to the properties of graphene plasmon, monolayer graphene exhibits tunable and strong nonlinear behavior. Various structures of nonlinear graphene and other 2D material have been summarized in Fig. 7, which can be categorized as doping graphene, graphene with metal/dielectric resonators, graphene integrated plasmon waveguide, structure with patterning graphene and structure with 2D TMDCs. For example, Mikhailov calculates the second order polarization rate and second order harmonic signal strength of graphene by self-consistent field method. As shown in Fig. 8, $\gamma / \omega_{\mathrm{p}}$ is the reciprocal of the quality factor of $2 \mathrm{D}$ plasmon resonance where $\gamma$ represents the momentum scattering rate, the smaller value represents the higher quality factor of the sample. The second harmonic radiation intensity increases several orders of magnitude with the condition of plasmon resonance [86]. In addition, graphene can achieve electrical control of nonlinear optical response due to gate tunability. Research groups from University of Cambridge, University of Milan and University of Genova first experimentally demonstrate the gate tunable THG based on graphene, which enable its applications for gate tunable switches and frequency converters. The research shows that the efficiency of THG can be controlled in the wide range of ultrawide bandwidth by external electric field control, and THG efficiency of graphene can be improved by more than 10 times 
[87]. However, since the absorption of graphene at optical frequencies is $2.3 \%$, which limits the enhancement of nonlinearity of graphene sheets. Several approaches have been designed to enhance the electric field by using the unique optical and nonlinear performance of graphene, including coupling graphene to metal/dielectric resonator, or integrating them into a light waveguide to effectively increase the length of the entire optical path. The fermi level of graphene can be tuned by chemical doping to adjust its dielectric constant, thereby controlling the nonlinear properties of graphene. In addition, patterned graphene can also promote a significant enhancement of the optical field.



Fig. 7. Various structure of nonlinear graphene and other 2D material. (a) Graphene with plasmon resonators. (b) Structure with doping graphene. (c) Graphene with patterning graphene (d) Structure with 2D TMDCs. (e) Structure with ENZ materials.

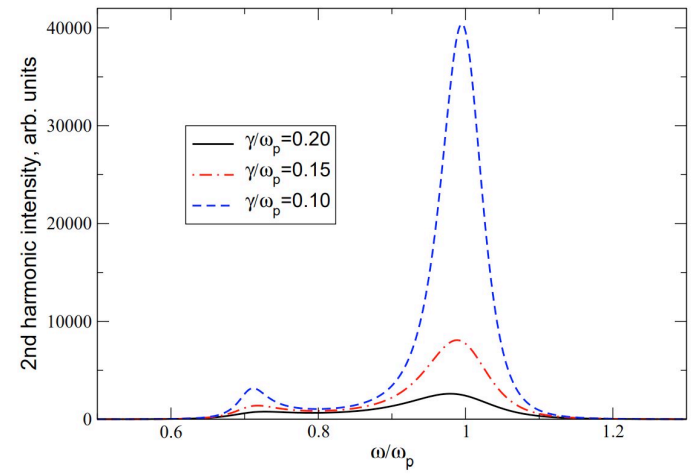

Fig. 8. Intensity of the second harmonic radiation in semiconductor structures and in graphene as a function of the frequency $\omega / \omega_{\mathrm{p}} \cdot \gamma / \omega_{\mathrm{p}}$ is the reciprocal of the quality factor of $2 \mathrm{D}$ plasmon resonance where $\gamma$ represents the momentum scattering rate, the small value represents the high quality of the sample. (S. A. Mikhailov). Reprinted with permission from [86]. Copyrights (2011) American Physical Society. 


\section{3a Graphene with plasmon resonator}

To further enhance the interaction between light and graphene, the most common method to enhance the nonlinearity of graphene is employing a plasmon resonator to enhance the local electric field. M. A. Vincenti et al. inserted the monolayer graphene into the defect layer of asymmetric one-dimensional photonic crystals which significantly enhance the THG. Once can see from Fig. 9 (a), The experimental results show that the narrow band absorption of graphene can reach 100\%. Fig. 9 (b) also demonstrated that even moderate electric field enhancement within periodic nanostructures is able to increase the THG by 5 orders of magnitude compared to that with only the pure graphene layer [88]. H. Zhou et al. use a slowlight silicon photonic crystal waveguide (PCW) shown in Fig. 9 (c) to enhance the FWM of single-layer graphene. The silicon photonic crystal nanomembrane with single graphene layer transferred on the substrate. Fig. 9 (d) shows the FWM results observed in the graphene-PCW (red solid), in comparison with the silicon-only PhCWG (blue dotted line). In this graphenesilicon slow-light hybrid configuration with an interaction length of $200 \mu \mathrm{m}$, a FWM conversion efficiency of $-23 \mathrm{~dB}$ has been achieved. Moreover, an enhanced 3-dB conversion bandwidth of $17 \mathrm{~nm}$ is obtained as well [89].


Fig. 9. Metal/dielectric resonators to enhance nonlinearity of graphene. (a) One-dimension photonic crystal structure with a graphene sheet placed in the center of the $\mathrm{SiO}_{2}$ (M. A. Vincenti et al.) [88]. (b) TH conversion efficiency of independent graphene (red dashed line) compared with that from photonic crystal structure (blue solid line) [88]. (c) The scanning electron microscope of the graphene $\mathrm{PhC}$ waveguide. The central area is continuously covered by monolayer graphene (H. Zhou et al.) [89]. (d) FWM results observed in the graphene-PCW (red solid), compared to the silicon-only PhCWG (blue dotted line) [89]. 


\section{3b Graphene by chemical doping or electric doping}

The change of Fermi level of graphene by chemical doping or electric doping leads to the change of its dielectric constant, so as to enhance and control the nonlinear properties of graphene. H. Wang et al. propose an Otto configuration composed of covering nonlinear substrates with multilayer graphene sheets to achieve tunable low-threshold optical bistability, as shown in Fig. 10 (a). Graphene was transferred on top of a nonlinear substrate. The substrate is separated from a prism made of germanium by a small air space. Graphene between the two media could support the single-bound surface plasmon mode, which is different from that propagating on the metal films. The p-type trans-conductive oxide $\mathrm{CuAlO}_{2}$ film was chosen as the electrode for the high conductivity and transparency in the terahertz range. Fig. 10 (b) shows the S-shaped curve of the relationship between the incident light intensity and the reflected light intensity. It is difficult to observe the hysteresis effect without graphene on the nonlinear substrate. However, by coating the nonlinear substrate with a single graphene sheet, the optical bistable behavior was observed immediately. Hysteresis in Fig. 10 (b) indicates the bistable state, which means that two output powers with high stability could be achieved with a fixed input power. Attributed to the strong local electric field enhancement from the surface plasmon excitation, the threshold of optical bistability was decreased by 1000 times. By precisely tuning the Fermi energy level of the graphene layer, the resonance transmission becomes stronger and the resonance peak moves significantly towards high frequency [90]. The strong interaction between light and graphene sheet can be further enhanced to reduce the power requirement of optical bistability and achieve tunable low threshold optical bistability at terahertz frequency [91]. A. V. Gorbach developes a perturbation expansion process of Maxwell equation and extends to graphene plasmon waveguide in Fig. 10 (c). The pulse propagation equation and the nonlinear coefficient of graphene are obtained. The Graphene surface plasmon waveguide through modulating the Fermi energy of the graphene layer is plotted in the upper part of Fig. 10 (c). The bottom of Fig. 10 (c) showed the electric field distribution at the fundamental guided mode. Fig. 10 (d) shows the output spectrum of a 100-second pulse propagating over the graphene surface plasmon waveguide. The results show that the characteristic nonlinear length is several orders of magnitude smaller than the damped propagation length, and the broadband spectrum covering more than one frequency octave can be obtained from the spectral broadening of $100 \mathrm{fs}$ pulse, with peak power of several hundred micro-watts and propagation distance of several hundred nanometers [92]. Joel D. Cox et al. utilize localized plasmon in doped graphene nanostructures to achieve the strong intensity required to produce high harmonics, as shown in Fig. 10(e). They demonstrate the synergistic effect between strong plasmon near- 
field enhancement and significant intrinsic nonlinearity and find that harmonics up to 13th order can be generated with a low light intensity of $100 \mathrm{MW} \mathrm{cm}$. The significant increase in high order harmonic generation has been shown in Fig. 10(f) where the emission intensity is mapped with input pulse carrier frequencies in a wide range. When the excitation frequency is coincident with the plasmon resonance, by passively tuning different wavelengths, the generated harmonics are obviously enhanced. [93].

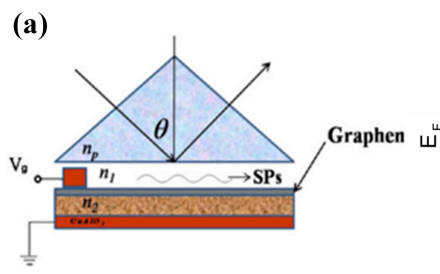

(c)
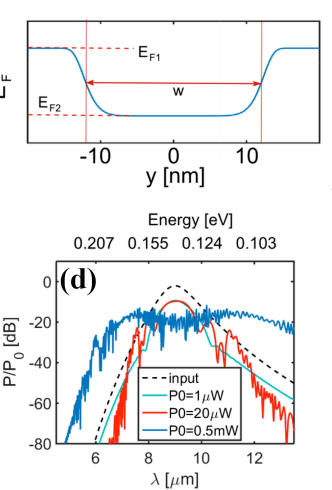

(e)

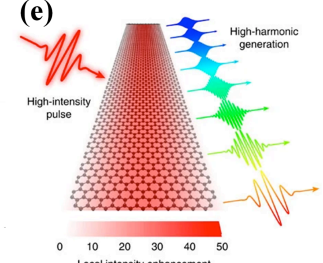

$\begin{array}{llllll}0 & 10 & 20 & 30 & 40 & 50\end{array}$

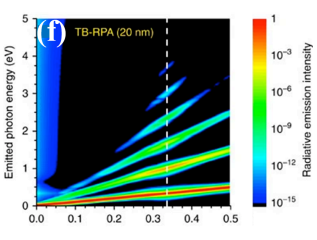

Fig. 10. Structures with doped graphene (a) Schematic representation of exciting SPs on Graphene via a gate voltage with the Otto configuration (H. Wang et al.) [90]. (b) Dependences of the reflectance and reflected light intensity on the incident light intensity. a typical s-shaped relation curve between incident and reflected light intensity illuminates optical bistability [90]. (c) Graphene surface plasmon waveguide formed by a modulation of the graphene Fermi energy, graphene is embedded into a dielectric (A. V. Gorbach) [92]. (d) The output spectrum of a pulse propagating over a graphene surface plasmon waveguide [92].

(e) Schematic illustration of a doped graphene nanoribbon illuminated by an optical pulse that is resonant with the graphene plasmon, producing strong in-plane electric-field enhancement that boosts the high harmonics generation (Joel D. Cox et al.) [93]. (f) The emission intensity from doped graphene nanoribbons under transverse normal illumination as a function of the incident and emitted photon energies [93].

\section{3c Patterned graphene}

An innovative design for enhancement and tuning optical fields is patterning graphene [94-96]. For example, M. Mohammad et al. proposed a theoretical model to measure the energy relaxation dynamics and terahertz nonlinear response of plasmon in the graphene nanoribbon. Fig. 11 (a) illustrates the dimensions and scanning-electron micrograph (SEM) images of the structure designed here, and Fig. 11 (b) compares the transmission for two different polarizations with the same incident pump frequency and fluence. The blue curve indicates that the polarization direction of the pump and probe was perpendicular to the graphene band, thus exciting the plasmon. The red curve represents the response under the 
same condition but with opposite polarization, there is no plasmon excitation at this point. The measured nonlinearity is much stronger when exciting plasmons than for the opposite polarization. Furthermore, the thermal model of nonlinear plasmon absorption further proposed shows that graphene with high mobility can obtain stronger nonlinear response [97]. H. Nasari et al. study the tunability of the resonant frequency of the ribbon graphene resonator. As shown in Fig. 11 (c), by changing the Fermi level of graphene or changing the refractive index of ribbon graphene on Kerr nonlinear substrate that can provide tunable bandpass filtering (Fig. 11 (d)). Through the cross-phase modulation process, the strength of the pumping signal can more effectively change the refractive index of ribbon graphene on the nonlinear substrate. The results show that the incorporation of Kerr nonlinear medium and ribbon graphene increases the tunability of the device, which provides a possibility for the application of all-optical tunable filter and ultra-fast switch [98]. F. Ling et al. employ periodic patterned graphene to achieve dynamic tunable plasmon-induced polarization conversion in the $\mathrm{THz}$ range. As exhibited in figure 11(e), the current of two vertical graphene dipoles and two connected T-shaped graphene monopoles is in the same phase at 2.3 THz frequency, which is caused by the radiation element directly excited by the incident terahertz wave. By tuning the Fermi level of T-type graphene through electrostatically controlling the voltage, the peak frequency and group index of the plasmon-induced transparency (PIT) adjusted, and the linear terahertz waves can be converted to elliptical polarization. In addition, by controlling the relaxation time of electrons in graphene, the elliptical polarization can be converted to right-handed circular polarization as a controllable polarization converter [99].


Fig. 11. Structures with pattern graphene (a) False color scanning electron micrograph of graphene nanoribbons (Mohammad m. et al.). Reprinted with permission from [97]. Copyrights (2016) American Chemical Society. (b) Comparison of the transmission for two different polarizations with the same incident pump fluence and frequency. The blue curve 


\begin{abstract}
indicates that the polarization direction of the pump and probe is perpendicular to the graphene band, thus exciting the plasmon. The red curve represents the response under the same condition but with opposite polarization, there is no plasmon excitation at this point [97]. (c) Schematic of ribbon graphene on Kerr nonlinear substrate (H. Nasari et al.). Reprinted with permission from [98]. Copyrights (2016) Optical Society of America. (d) Tunability of the proposed band-pass filter by altering the graphene Fermi level [98]. (e) Schematic diagram of the structure of plasmon induced polarization conversion composed of two graphene cutting lines and two connected t-shaped graphene bands (F. Ling et al.). Reprinted with permission from [99]. Copyrights (2016) Springer Nature. (f) Electric field distribution and surface current distribution (red arrows) [99].
\end{abstract}

\title{
2.3d Structures with 2D TMDCs materials
}

In addition to graphene, the 2D TMDCs have attracted attention recently due to the remarkable electrical and optical properties, especially the broken inversion symmetry in their single-layer. Monolayer 2D TMDCs are usually consisted of three atomic layers stacked through van der Waals forces. K. L. Seyler et al. combined the plasmonic metasurface with the cavity filled with $\mathrm{WSe}_{2}$ to electrical control SHG effect, as shown in Fig. 12 (a). The structure consists of $\mathrm{WSe}_{2}$ crystals on $\mathrm{SiO}_{2}$ and $\mathrm{n}+$-doped $\mathrm{Si}$, with $\mathrm{V} / \mathrm{Au}$ contacts. The $\mathrm{SHG}$ is produced by third-order nonlinear optical effects including fundamental waves and a static field. The SHG efficiency can be regulated by controlling the electrostatic doping. There is a $\sim 4$ times of reduction in the SHG intensity peak when the gate voltage varies from -80 to 80 $\mathrm{V}$ (Fig. 12 (b)). The intensity of SHG at the resonance can be tuned to a 10 times enhancement at low temperature and 4 times improvement at room temperature based on the electrostatic doping within a field-effect transistor device. This metasurface provides a guideline for future development of on-chip nonlinear optical device based on 2D semiconductors with atomically thin layers [100]. X. Lin et al. proposed a pyramid-like multilayer $\mathrm{WS}_{2}$ structure, as shown in Fig. 12 (c). Because the two layers of each cell are stacked symmetrically in a hexagonal structure, the second-order nonlinear polarization directions of the adjacent layers are opposite when excited by nonlinear polarized laser, resulting in the local destructive interference of the $\mathrm{SH}$ field, the multilayer $\mathrm{WS}_{2}$ structure could have effective edge SH radiation, as shown in Fig. 12 (d). In addition, with the increasing number of atomic layers, the interaction length between light and matter in multilayer TMDCs increases. Through this significant improvement with more effective light confinement, a whispering-gallery mode resonance is generated. Compared with single-layer $\mathrm{WS}_{2}$ under the same conditions, the SHG of the multilayer TMDCs structure is enhanced by more than 40 times. Moreover, by coupling the whispering-gallery mode to the plasmonic mode, a SHG enhancement factor of 800 has been achieved [101]. D. Li et al. designed a hybrid structure of $\mathrm{MoS}_{2} / \mathrm{TiO}_{2}$ nanowires, which significantly improved the nonlinear optical 
conversion efficiency. The designed hybrid structure is shown in Fig. 12 (e). $\mathrm{TiO}_{2}$ nanowires suspension is spin-coated on a clean silica/silicon substrate. After drying, the detached monolayers of silicon dioxide are transferred to the selected titanium dioxide nanowires. SHG enhancement is due to strong light coupling and the suspension of the $\mathrm{MoS}_{2}$ without the substrate-induced doping and dielectric screening effects. The lattice deformation induced by one-dimensional nanowires is the key factor for the enhancement of strongly anisotropic SHG in atomic lamellar $\mathrm{MoS}_{2}$. Fig. 12 (f) shows the excitation power-dependent SHG of single $\mathrm{MoS}_{2}$ without and with $\mathrm{TiO}_{2}$ nanowires. The expected quadratic power dependence of the SHG signal from single $\mathrm{MoS}_{2}$ further confirms the second-order nonlinear light emission [102]. Plasmon modulators are devices that control the amplitude or phase of the propagating surface plasmon. It can be achieved by tuning the interaction between different plasmon waves. M. Klein et al. showed a 2D semiconductor nonlinear plasmon modulator based on $\mathrm{WSe}_{2}$ monolayer integrated on a metal waveguide [103]. The schematic diagram of the structure is shown in Fig. 12 (g), the free space laser can excite the SPP at the input of the device through the coupler. SPP propagates through the waveguide and interacts with excitons in the active $\mathrm{WSe}_{2}$ encapsulated in hBN. Finally, the SPP is coupled back to free space photons through the output grating coupler. The light transmission of plasmon devices could be controlled by optically pumping $\mathrm{WSe}_{2}$ excitons and partial absorption. The SPP propagating on the waveguide served as a probe, and the free-space laser focused on $\mathrm{WSe}_{2}$ is used as an optical pump. The time resolved DT/T response, which is the pump-induced differential transmittance (DT) normalized by the probe transmittance (T), is plotted in Fig. 12 (h), fast $290 \pm 20$ fs and slower $13.7 \pm 0.6$ ps components to the decay time are achieved, which is 5-10 times faster than previous work for monolayer $\mathrm{WSe}_{2}$ on $\mathrm{SiO}_{2}$ [104].

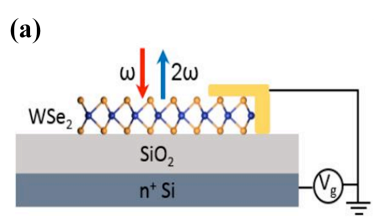

(b)

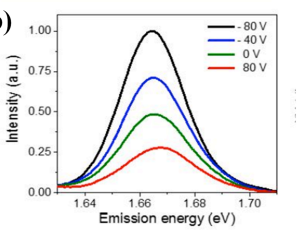

(c)
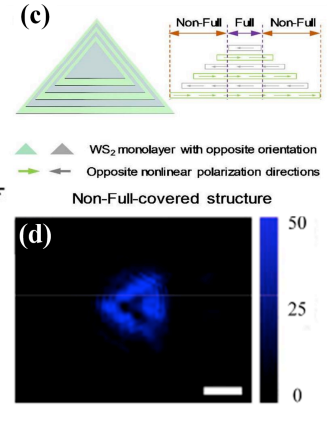
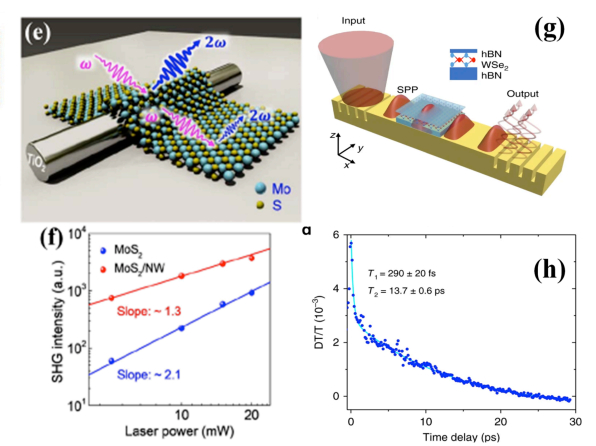

Fig. 12. (a) Second-harmonic generation in gated monolayer $\mathrm{WSe}_{2}$. red arrow indicates the excitation at $\omega$, and blue arrow respects second-harmonic radiation at $2 \omega$ (K. L. Seyler et al.). Reprinted with permission from [100]. Copyrights (2015) Springer Nature. (b) SHG spectra on resonance with exciton at selected gate voltages [100]. (c) Schematic diagram of a pyramid- 
like $\mathrm{WS}_{2}$ structure where the second-order nonlinear polarization directions of the adjacent layers are opposite (X. Lin et al.) [101]. (d) SH optical microscopy images of a multilayer $\mathrm{WS}_{2}$ structure [101]. (e) Schematic diagram of single $\mathrm{TiO}_{2}$ nanowire integrated monolayer $\mathrm{MoS}_{2}$, the radiation frequency of fundamental wave radiation frequency $\omega$ (D. Li et al.) [102]. (f) The SHG intensity excited by monolayer $\mathrm{MoS}_{2}$ and $\mathrm{MoS}_{2} / \mathrm{NW}$ hybridization as a function of the excitation laser power [102]. (g) Nonlinear plasmon modulator based on $\mathrm{WSe}_{2}$ monolayer integrated on a metal waveguide. SPPs are excited at the input of the device, propagate through the waveguide and interacts with $\mathrm{WSe}_{2}$ (M. Klein et al.) [104]. (h) Time resolved DT/T response, which is the pump-induced differential transmittance normalized by the probe transmittance, shows 5-10 times faster decay time than previous work [104].

\section{3e Structure with ENZ dielectric materials}

ENZ dielectric is obtained by designing metallic dielectric materials properly that exhibits a near zero dielectric constant [105]. It has been proposed that ENZ materials have characteristics of controlling the propagation and positioning of electro-magnetic field. In addition, the enhancement of the normal component of electric field in epsilon near-zero medium can be predicted theoretically, and has become a practical strategy to enhance the generation of nonlinear harmonics and could also improve the optical bistability of Kerr nonlinear devices [106-108]. For example, B. Wells et al. obtain a strong second-order nonlinear response in plasmonic nanorods composed of gold nanoparticles plating on nanoporous anodized aluminum. An effective-medium theory was developed and the limitations of its applicability are analyzed. Under the limitation of small absorption, long nanorods or large unit cells, the error of local effective-medium theory prediction could be quantitatively explained by incorporating the delay effect into non-local effective-medium theory $[109,110]$. The structure of the designed composite is shown in Fig. 13 (a). The results show the generation of strongly tunable p-polarized second harmonic light that corresponds to s-polarized or p-polarized excitation, respectively (Fig. 13 (b)). For the fundamental frequencies of the groups under the ENZ spectral range, the enhancement of the second harmonic is observed. The absorption of materials in gold plays an important part in the SHG process in ENZ scheme, reducing the loss of gold, thus increasing the efficiency of SHG in metamaterials by an order of magnitude. This work reshapes the electromagnetic fields in metamaterials using plasmon components, which can be used to transform SHG from surface to volume-dominated regions, and to design strongly tunable volumetric nonlinear responses in plasmon composites. The proposed plasmon nanorod SHG method can be also used to analyze the nonlinear responses of metasurfaces consisting of composite materials [111]. In recent years, transparent conducting oxide, for example, indium tin oxide (ITO) and nitrogenbased nanofilms have received extensive attention as alternative candidates for plasmonic metamaterials due to its lower loss both in the near infrared and visible spectrum, and their 
toleration in high temperature and electric fields. Because the resulted dielectric constant at the wavelength of the plasmon is zero, it has a broad application prospect in nonlinear optics $[112,113]$. For example, N. Kinsey et al. reported an epsilon-near-zero AZO film by employing zinc oxide doped with aluminum (AZO), which simultaneously exhibited hypervelocity carrier dynamics and excellent reflectance modulation for low pump levels at $1.3 \mu \mathrm{m}$ [114]. A. Capretti et al. demonstrated the third harmonic generation of ITO nanocrystal lines, which is about 600-fold more efficient than crystalline silicon [113]. Fig. 13 (c) and (d) shows the intensity dependence index of ITO under transverse magnetic (TM)-polarized light characterized by z-scan technique [115]. The effective nonlinear attenuation constant and effective nonlinear refractive index coefficient as a function of incident wavelength were extracted with incident angle at $0^{\circ}$ and $60^{\circ}$. The results showed that ITO is respectively positive when self-focusing, and negative when saturated absorption. The value of $n_{2 \text { (eff) }}=$ $0.11 \mathrm{~cm}^{2} / \mathrm{GW}$ measured with incident angle at $60^{\circ}$ is two orders of magnitude larger than that of $\mathrm{As}_{2} \mathrm{Se}_{3}$ chalcogenide glass [116], and is 5 times higher than the recently reported nonlinear metamaterial [117]. As a result, the effective nonlinear attenuation constant and refractive index coefficient and at the incident wavelength of $1240 \mathrm{~nm}$ were $\sim 53$ and $\sim 43$ times higher than those with $970 \mathrm{~nm}$.
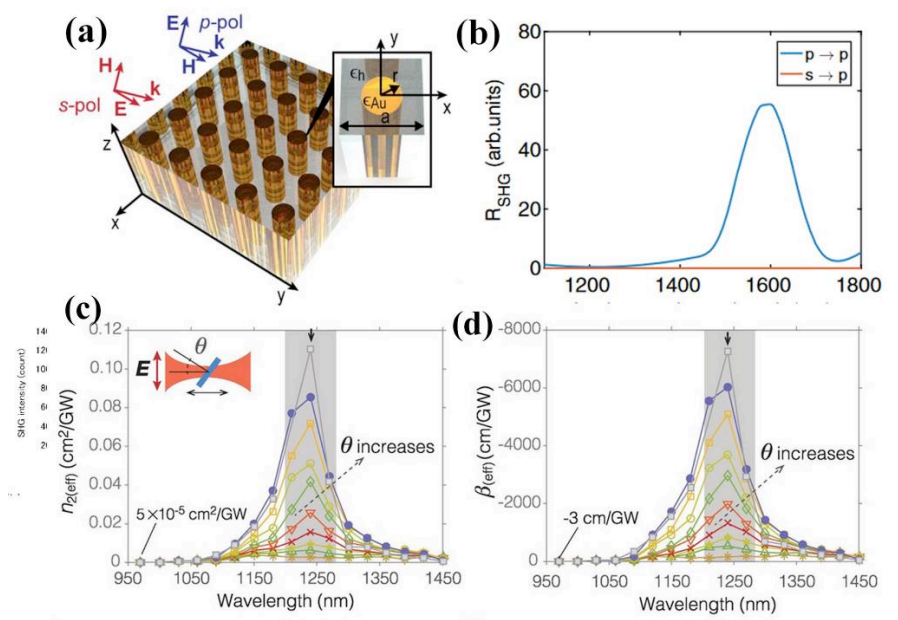

Fig. 13. (a) Proposed schematic geometry of metamaterial and directions of fields and wavevectors (B. Wells et al.). Reprinted with permission from [111]. Copyrights (2018) Optical Society of America. (b) SHG spectra with s $\rightarrow$ p SHG [111]. Wavelength dependence of the (c) nonlinear effective refractive index and (d) effective nonlinear attenuation constant as a function of wavelength. The nonlinear response is enhanced in the ENZ region of the spectrum (shaded) (A. Capretti et al.)[115]. 


\section{Nonlinear metasurfaces}

Benefiting from the ability to enhance light-matter interactions and control local optical polarization, metasurfaces are employed to engineer the nonlinear optical effects. Especially in the terahertz $(\mathrm{THz})$ frequency, the concentration of incident $\mathrm{THz}$ waves relaxes the requirements of high field intensity, making it possible to generate high power $\mathrm{THz}$ radiation. Various nonlinear parameters including intensity, phase, and polarization states, can be controlled effectively by changing the shape anisotropy and geometry of metasurfaces. It provides an effective manipulation for nonlinear interaction. In particular, nonlinear metasurfaces based on plasmonic nanostructures are the most appropriate candidates for improving the nonlinear optical response because of the strong local electromagnetic field enhancement caused by plasmonic resonant, which increases the pump efficiency significantly. Various types of metasurfaces for enhanced nonlinear optical response are summarized in Fig. 14, which can be generally categorized by arrays of non-centrosymmetric metal nanoparticles, arrays of anisotropic elements or different kinds of elements, arrays of semiconductor materials, Fano resonance based metasurfaces, plasmonic metasurface coupling to the quantized electron sub-bands and patterned nonlinear waveguides coated with phase gradient metasurfaces.

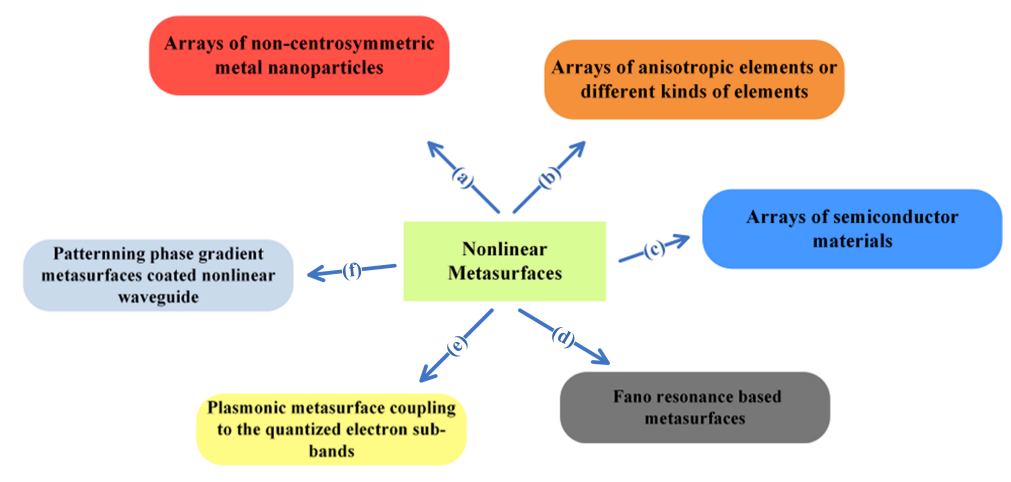

Fig. 14. Different structure of nonlinear metasurfaces. (a) Arrays of non-centrosymmetric metal nanoparticles. (b) Arrays of anisotropic elements or different kinds of elements. (c) Arrays of semiconductor materials. (d) Fano resonance based metasurfaces. (e) Plasmonic metasurface coupling to the quantized electron sub-bands. (f) Patterned nonlinear waveguide coated with phase gradient metasurfaces.

\subsection{Arrays of non-centrosymmetric metal nanoparticle}

So far, some research groups have experimentally studied the nonlinear characteristics of metal array. Plasmon metasurfaces are based on meta-atoms made of metal nanostructures, where their optical responses are dominated by the plasmon resonances of the nanostructure. Effective electrical or magnetic polarizability is defined by properties such as particle 
material and geometry [118]. G. Vampa et al. demonstrated the use of arrays based on plasmonic monopole antennas to achieve high harmonic generation with crystalline silicon substrate. The scanning electron micrograph of monopole Au nano-antenna arrays are plotted in Fig. 15(a). Contributing to the plasmon from the antenna arrays, the highly density substrate allowed the accumulation of harmonic emission under the microscope, which was ten-fold brighter than that without the plasmonic antennas, as plotted in Fig. 15(b). The polarization of the harmonic beam in the near field could be tuned by using the harmonic sensitivity to the incident linear light polarization at the main axis of the antenna. More precise harmonic beam control could be extended to the corresponding structures of these metasurfaces [119]. B. k. Canfield et al. introduced the sensitivity of the optical response to the polarization state of the incident beam in a low-symmetric L-shaped gold nanoparticle array [120]. Fig .15(c) shows the ideal "L" particle, the symmetry of its main axis (expressed as $\mathrm{X}$ and $\mathrm{Y}$ ) implies the natural selection of the main axis. Fig. 15(d) shows the normalized SHG polarization responses for XY polarizations, clearly demonstrates the response of SH to the symmetry breaking in the samples. Prohibiting parallel response YYY is not only nonzero, but also reaches a considerable proportion of the maximum response (YYY is about $10 \%$ of XYY). In addition, these results indicate that, in conjunction with the rotation of the polarization azimuth, the nonlinear measurement will produce other significant information about the symmetry breaking. The experimental results showed that the break of symmetry led to the large angular displacement of the extinction axis, and the optical response of the array became sensitive to the polarization state of the incident beam. The concept of Pancharatnam-Berry phase was first proposed in linear metasurface, An optical element based on Pancharatnam-Berry phase can introduce the geometric phase difference of transmitted (or reflected) waves according to its geometric shape and direction [121]. By gradually changing the local direction of each unit, the local transmission or reflected waves can be effectively adjusted [122]. Z. Li et al. extended the Pancharatnam-Berry phase method to nonlinear optics, designed metasurfaces with huge nonlinear responses, and enriched their functions. The nonlinear metasurfaces consisting of anisotropic U-shaped metal nanoparticles which introduced the second harmonic pancharatnami-berry phase [121]. The proposed structure was shown in Fig. 15(e). Here, optical vortices with different topological charges could focus on various focal planes under different spin and wavelength combinations. They also propose a simplified interferometry using a \pm 1 order phase mask to determine the topological charge of the optical vortex with an ultrafast laser source. In this way, the zero-order and maximum are suppressed and light could be equal diffracted to \pm 1 order, as shown in Fig. 15(f). The self-interference between the two orders produces a phase mask interference pattern of half 
period, which results in a pair of fringe pattern dislocations in the optical vortex interference pattern. More importantly, three branches $(-\sigma)$ and one branch $(\sigma)$ have also been observed in the fringe patterns. This phenomenon demonstrated the phase defects existed at the cores of the two SHG beams. Compared with the conventional linear metasurfaces, this structure could simultaneously produce three times capacity of optical turbines, including two SHG optical vortices and one linear vortice, with topological charges focused at different focal lengths. In addition, the capacity of optical turbines could be further expanded by introducing other nonlinear processes such as THG. These flexible features of this nonlinear optical vortex generation process could be utilized for enhancing the conventional optical communication and multi-channel integrated optical communication systems [123].
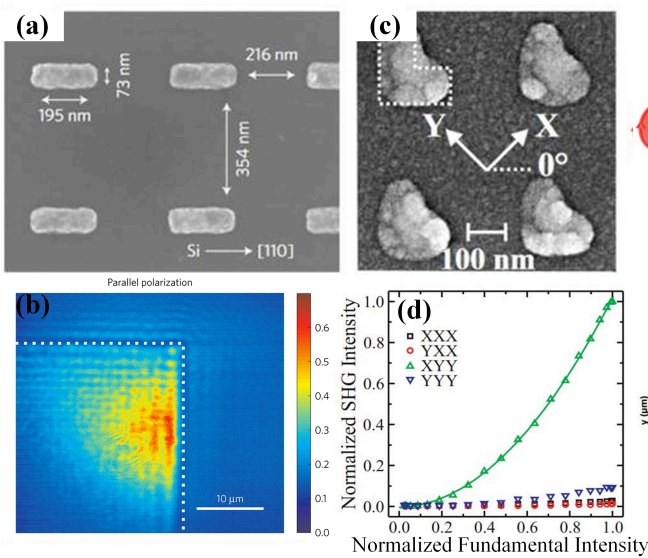

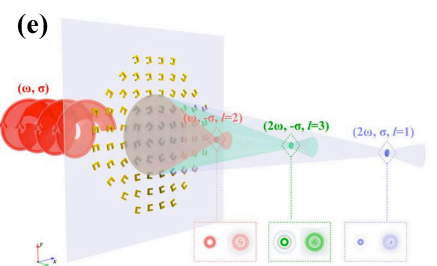

(f)
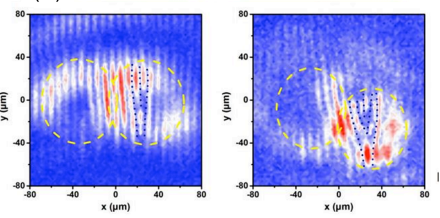

Fig. 15. Nonlinear metasurfaces consist of arrays of non-centrosymmetric metal nanoparticles.

(a) Scanning electron micrograph of monopole $\mathrm{Au}$ nano-antenna arrays. Design of metasurfaces with anisotropic elements or different kinds of elements (G. Vampa et al.) [119].

(b) Image with the 5th harmonic of a corner of an illuminated array [119]. (c) Scanning electron micrograph of the L-shape nanoparticles (B. k. Canfield et al.). Reprinted with permission from [120]. Copyrights (2004) Optical Society of America. (d) Normalized SHG polarization responses for selected combinations of XY polarizations [120]. (e) A schematic diagram of metasurface focusing optical vortices. Three optical vortices with different topological charges focus on different focal planes (Z. Li et al.). Reprinted with permission from [123]. Copyrights (2018) WILEY-VCH Verlag GmbH \& Co. KGaA, Weinheim. (f) Experimental measurement of the topological charges of SHG optical vortices [123].

\subsection{Arrays of semiconductor materials}

More recently, semiconductor nanomaterials such as $\mathrm{Si}$ and GaAs semiconductors with high refractive index have proven to be able to significantly enhance the plasmon properties, which can be used for photodetection and nonlinear optics [124-126]. The plasmon metasurface integrated with semiconductor materials has been proposed and widely used in 
nonlinear optics. P. P. Vabishchevich et al. report the enhanced SHG in hybrid metasurface made up of Fano structure and asymmetric GaAs nano-disk metasurface, as exhibited in Fig. 16(a). The shape of the nano-resonator leads to a pattern coupling between the transverse and longitudinal dipole modes of the perpendicular incident light, resulting in Fano resonance with a high Q value. Fig. 16(b) shows the SH signal intensity generated when the pump wavelength sweeps over the metasurface with the pump power unchanged. The SH intensity spectrum exhibits a narrow peak at the Fano resonance wavelength. As the local electromagnetic field is significantly enhanced at the resonance peak, SHG signal is 300 times higher than off-resonant when the pump laser is tuned to Fano resonance [127]. Mie dielectric resonators attract several attentions in nonlinear optics due to their low intrinsic loss [128, 129], dielectric metasurfaces with semiconductors contribute to the excitation of intrinsic second order nonlinearity and enhance the generation of harmonics. S. Liu et al. demonstrate dielectric metasurfaces made up of GaAs resonator arrays as exhibited in Fig. 16(c). Fig. 16(d) shows the SHG intensity when the pump power remains unchanged. With the strong enhancement of the electromagnetic field excited at these resonances, the SHG power reaches at peaks near the magnetic and electrical dipole resonances. When the pump is coincident with the magnetic dipole and the electric dipole, the SH signal is 3 and 1 orders of magnitude improved in comparison to the signal obtained when the pump is separated from the cavity length. The experiment also shows that operating at longer wavelengths can reduce GaAs absorption at the SH wavelength, and lead to enhancement of the SH field within the resonator, thus achieve higher conversion efficiency [130].

(a)


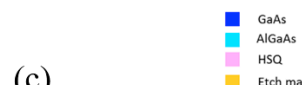

(c)



(d)

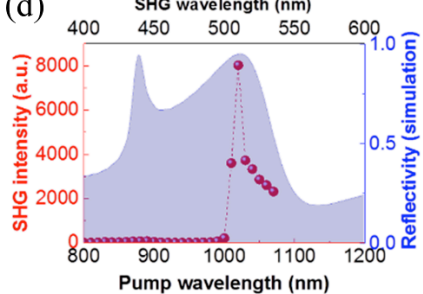

Fig. 16. Semiconductor materials in nonlinear optics. (a) Schematic diagram of high-Q Fano resonances GaAs metasurface, the element is a cube that has a notch cut from the corner (P. p. 


\begin{abstract}
Vabishchevich et al.). Reprinted with permission from [127]. Copyrights (2018) American Chemical Society. (b) Experimental results of the SHG intensity spectrum, showing the resonantly enhanced SHG at the magnetic dipole resonance. Reflectivity spectrum of the nanodisc metasurface are used as the backgrounds. Inset: top-view SEM image of a nanodisc metasurface sample [127]. (c) Proposed GaAs based dielectric metasurfaces, consists of a square lattice of GaAs nanodisk resonators lying on a $\left(\mathrm{Al}_{\mathrm{x}} \mathrm{Ga}_{1-\mathrm{x}}\right)_{2} \mathrm{O}_{3}$ native oxide spacer layer (S. Liu et al). Reprinted with permission from [130]. Copyrights (2016) American Chemical Society. (d) Results for the spectral dependence of the SHG intensity. The linear reflectivity spectra of the sample are used as the backgrounds [130].
\end{abstract}

\title{
3.3. Fano resonance based metasurfaces
}

The high loss caused by plasmonic resonance severely limits the spectral selectivity of the vast majority of metasurfaces made up of metals. In this context, metasurface based on electromagnetic-induced transparency (EIT) and Fano resonances become the focus of the field of nanophotonics, as they can generate high quality factor resonance. Metasurface consisting of CMOS compatible materials, silicon and silicon oxides are proposed by $\mathrm{C} . \mathrm{Wu}$ et al. The conceptual schematic diagram of the geometric structure of the unit cell used here was demonstrated in Fig. 17 (a). Each cell consists of a straight and a bent silicon nanorod. And the bent nanorod is used here for breaking the two mirror inversion symmetries of the unit cell. In Fig. 17 (b), one can see the coupling effect of the dark (magnetic dipole) and bright (electric dipole) resonators. The surface charge density of between the air and silicon interface was shown when the eigenmodes of the element surface with and without symmetric fracture bending. The proposed metasurface is suitable for various applications including IR spectra detection of biological and chemical markers and for other improved nonlinear optical devices [131]. Combining the high field intensity in this silicon metasurface with a coherent radiation source can also be used to enhance nonlinear effects including harmonic generation and FWM $[132,133]$. Y. Yang et al. employed silicon metasurface based on Fano resonance to enhance third-order nonlinearity, which is used to improve THG efficiency. The metasurface based on Fano resonance is shown in Fig. 17 (c). The unit cell has a periodic lattice consisting of a coupled rectangular bar and a disk resonator based on silicon. These rectangular bar resonators could induce a "bright" electric dipole resonance excited with an incident electric field along the $\mathrm{X}$-axis whereas the disks generated a "dark" magnetic dipole resonance. Due to the high $\mathrm{Q}$ factor provided by Fano resonance, the local electric field in $\mathrm{Si}$ is strongly enhanced, resulting in a strong near-field enhancement of the metasurface in the volume of the silicon resonator. Benefiting from the characteristics of the ring resonator, there is no bus waveguide, which can minimize the two-photon absorption. Fig. 17 (d) simultaneously measures the pump light power and the third harmonic power and 
characterizes the absolute third harmonic efficiency of the metasurfaces based on Fano resonance. According to these measurement results, when the average pump power was at a value of $50 \mathrm{~mW}$ and the peak pumping intensity was $3.2 \mathrm{GW} \mathrm{cm}^{-2}$, the conversion efficiency was $1.2 \times 10^{-6}$, which was the highest reported to date in other comparable pump energies [129].
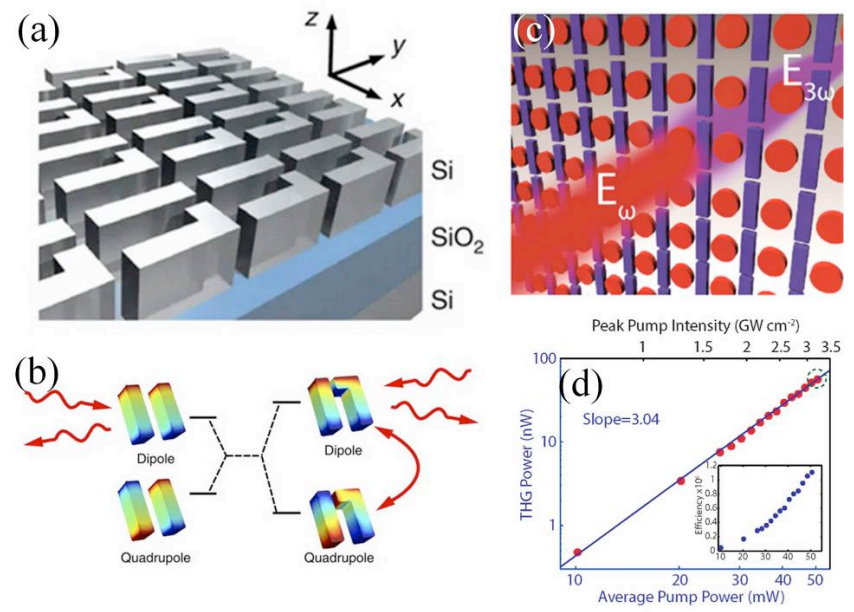

Fig. 17. FR based construction for improvement of nonlinear effects. (a) A schematic of the silicic metasurface with high-Q FRs, each cell consists of a straight and a bent silicon nanorod, and the bent nanorod is used here for breaking the two mirror inversion symmetries of the unit cell (C. Wu et al.). Reprinted with permission from [131]. Copyrights (2013) American Chemical Society. (b) A schematic illustrates the electric dipole (upper left) and quadrupole (lower left) modes in Fano interference [131]. (c) Schematic of silicon metasurface based on Fano resonance. The bar resonators represent the "bright"mode, and the disk resonators represent the“dark"mode (Y. Yang et al.). Reprinted with permission from [129]. Copyrights (2015) American Chemical Society. (d) Graph of the third harmonic power as a function of the pump power and the peak pump intensity. The red circles are the measured data, and the blue line is a numerical fit to the data. The inset is the absolute THG efficiency as a function of the pump power [129].

\subsection{Plasmonic metasurfaces coupled to intersubband transitions}

The strong coupling of resonators to intersubband transitions had been studied and recently demonstrated [134, 135], which means that the device can take advantage of the giant nonlinearity provided by intersubband transitions. A. Benz et al. presented experimental evidence of strong optical coupling between single subwavelength nano-metamaterial and an intersubband transitions in semiconductor heterostructure. The schematic diagram of the experiment is shown in Fig.18(a). The metamaterial resonator is fabricated on the semiconductor substrate. The light is incident vertically into the sample and polarized along 
the Y-axis. When intersubband transitions and resonators are coupled with each other, two separate resonances are coupled into two polarized branches, as demonstrated in the inset of Fig.18(a). The interaction between strong light and matter was studied by normal incident light transmission spectrum, as plotted in Fig.18(b). When the resonance frequency of the metamaterial changes across intersubband transitions resonance, the transmission spectrum shows two polarization branches with significant anti-crossover behavior. In the case of offresonance, a unique minimum associated with metamaterial resonator was obtained along with a weaker minimum associated with intersubband transitions. When two resonances become similar in the energy, the weights of the two minimum values would be comparable [136]. Inspired by them, O. Wolf et al. achieved complete control of the polarization of the emitted second harmonic signal by a double-resonant nanometer array near-field coupled to interband transition with highly nonlinearity in a semiconductor quantum well. The combination of the quantum well layer that produces second harmonics and the resonator that controls the beam is depicted in Fig. 18(c), coherent emission from a single resonator provides the possibility of different beam shaping. Through the relationship between the measured second harmonics power and the basic power $(10.22 \mu \mathrm{m})$ in the inset of Fig.18(d), they obtained the second harmonics conversion efficiency of $0.45 \mathrm{mWW}^{-2}$. To prove that the spectral response depends on both the resonator and the quantum well, the conversion efficiency is plotted as a function of the pump wavelength in Fig.18(d). The data showed that the efficiency was strongly dependent on the wavelength, and the maximum conversion efficiency was as high as $2.3 \mathrm{mWW}^{-2}$, which was the highest value as reported in the intersubband transitions reorganization structure [137]. Y. Todorov et al. realized a nonlinear metasurface based on the electromagnetic mode in the plasmonic surface coupling to the quantized electron sub-bands in the semiconductor heterostructure, as shown in Fig. 18 (e). A multi-quantum-well layer was sandwiched between a metallic substrate and metallic arrays. Effective coupling occurred for the plasmonic mode. The z-polarized intersubband transitions in this grounded metasurfaces strongly enhanced the interaction between light and matter, and converted the cross-field polarization of the collision into the desired $\mathrm{z}$ direction [138]. Because the nonlinear effect is limited to the condition that the electric field of incident light is perpendicular to the polarization of semiconductor layer, coupling with plasmonic metasurface improves the light-matter interaction strongly and produces exotic wave properties. Experiments show that nonlinear susceptibility tensor for these nanostructures could be designed by implementing a metasurface with nonlinear susceptibility of $>5 \times 10^{4}$ $\mathrm{pm} / \mathrm{V}$ for SHG with an excitation wavelength of 8 um under normal incidence, which is one of the highest susceptibilities in optical metasurfaces measured in current literatures [139- 
141]. Fig.18 (f) shows the SHG emission spectra of the metasurface under different fundamental frequencies. Multi-quantum-well structure without nanostructure has not shown any nonlinear response to normal incident light, as shown in the insert figure. The proposed structures are able to achieve effective frequency mixing under relaxed phase matching conditions, and also allow broadband frequency up- and down-conversion [142] where alloptical control and the phase conjugation are demonstrated as well. The metasurface based on Pancharatnam-Berry phase optical elements discussed in Section 3.1 can introduce geometric phase difference between transmitted (or reflected) waves according to its geometric shape and direction. Recently, M. Tymchenko et al. extended the Pancharatnam-Berry phase optical element to the multi-quantum-well-semiconductor-based nonlinear plasmon metasurface to adjust the spatial phase distribution of its effective SH radiation. The proposed nonlinear Pancharatnam-Berry metasurface is shown in Fig.18 (g). A thin multi-quantum-well substrate is sandwiched between a properly designed plasmon resonator array and a metal ground plane. The incident beam propagates in the $\mathrm{z}$ direction, and the metasurface operates in reflection. Each element is designed to have a large second harmonic conversion efficiency, and rotated to obtain the local geometric phase required for circularly polarized incidence. More importantly, these large nonlinear effects are very sensitive to the change of local resonance on the surface. Thus, the method based on Pancharatnam-Berry phase becomes an ideal tool to realize complete phase control. Fig.18 (h) shows the phase of the effective induced surface currents and the corresponding standardized tensor elements under right-handed and lefthanded circularly polarized fields, respectively. The results are in the same order of magnitude as those without Pancharatnam-Berry phase method, which proves that the phase control function does not affect the nonlinear process. Therefore, the Pancharatnam-Berry phase method can greatly enrich the functions of the metasurface with giant nonlinear response [121].
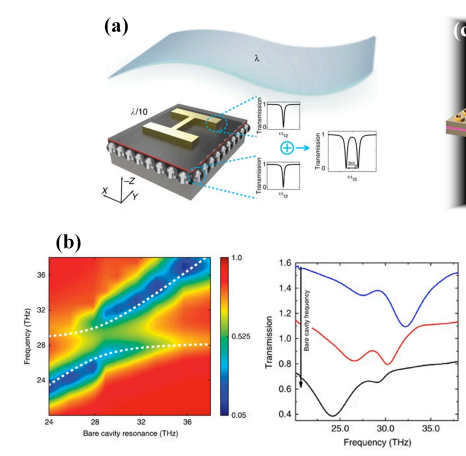

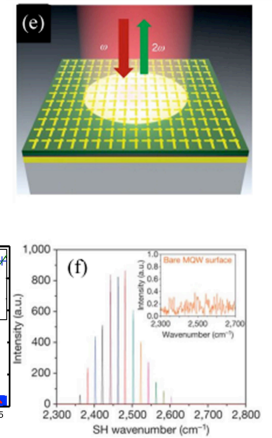
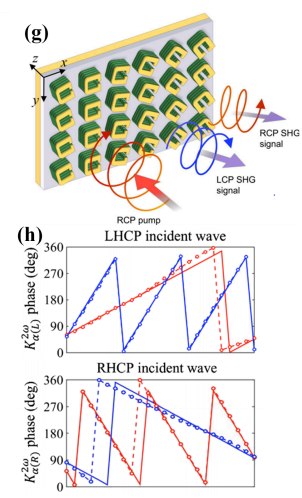
Fig. 18. Design of plasmonic metasurfaces coupled to intersubband transitions. (a) The metamaterial resonator is fabricated on the semiconductor substrate. The light is incident vertically into the sample and polarized along the Y-axis. When intersubband transitions and metamaterial resonators are coupled with each other, two separate resonances are coupled into two polarized branches (A. Benz et al.) [136]. (b) The simulated transmission spectrum of proposed structure [136]. (c) The combination of the quantum well layer that produces second harmonics and the metamaterial resonator that controls the beam. Phase-coherent emission from a single resonator opens the door to many beamforming applications, which are presented in insets (O. Wolf et al.) [137]. (d) The conversion efficiency as a function of the pump wavelength, the inset shows the relationship between the measured second harmonics power and the basic power [137]. (e) Schematic of the proposed metasurface, red and green arrows indicate the excited pump light at $\omega$, and generates second-harmonic radiation at $2 \omega$, respectively (Y. Todorov et al.). Reprinted with permission from [138]. Copyrights (2014) Spring Nature. (f) Spectra of SH output for different pump wavenumbers. Inset, no SH emission is observable for a bare MQW surface with no nanostructures on it [138]. (g) The schematic diagram of the mixing device. The metasurface consists of a guiding layer with high dielectric constant, and a dielectric grating on it. Multiple coupled quantum well designs can provide optical conversions at both the basic frequency and the second harmonic frequency (R. Sarma et al.) [143]. (h) Calculated reflectance from the structure at normal incidence [143].

\subsection{Nonlinear waveguides coated with phase gradient metasurfaces}

In recent years, metasurfaces with the ability of control the propagation of light had been designed to control guided waves and its coupling with free light. Z. Li et al. achieved waveguide mode converters and polarization rotators through plasmonic gradient metasurfaces for the mid-infrared region. As plotted in Fig. 19 (a), the gold-array antennas were patterned on silicon waveguides with resonance wavelength centered at $4 \mu \mathrm{m}$. Along the plasmon propagation direction, the fundamental waveguide mode is coupled into higher-order modes, as shown in Fig. 19 (b). A unidirectional wave generator on the gradient metasurfaces was employed to facilate the phase matching for nonlinear wavelength conversion [144]. Similarly, they also demonstrate a nonlinear waveguide with patterning phase gradient metasurfaces at the top layer to realize nonlinear wavelength conversion. The collective effect of the phase nano-arrays of the gradient metasurfaces contributed to the large volume of nonlinear materials to improve the nonlinear generation efficiency and therefore broke the symmetry of the coupling between the nonlinear signal and the pump signal. This design allows the nonlinear generation processes to maintain high efficiencies for a wide range of pumping wavelengths. Integrated nonlinear metasurfaces consisting of gradient metasurfaces and $\mathrm{LiNbO}_{3}$ waveguide were plotted in Fg. 19(c). The gradient metasurface, consisting of dielectric phased antenna arrays, is patterned on the top surface of a nonlinear optical waveguide. Fig. 19(d) shows the working mechanism of the proposed device. Soon as the 
optical power coupled from the fundamental mode at pump frequency to that at the $\mathrm{SH}$ frequency, the mode started to convert to the higher-order waveguide mode at the $\mathrm{SH}$ frequency. The unidirectional wave vector of the gradient metasurfaces preventes the light power from being back to the fundamental mode once reached to the higher order of $\mathrm{SH}$ frequency. The results showed several times of enhancement in nonlinear wavelength conversion efficiency [145].
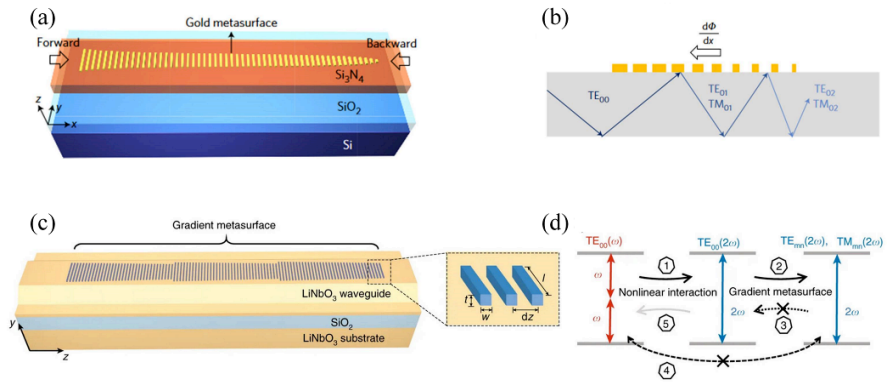

Fig. 19. (a) Schematic of metasurfaces consists of gold nanorods with different lengths located on the top of $\mathrm{a} \mathrm{Si}_{3} \mathrm{~N}_{4}$ waveguide that supports highly asymmetric optical power transmission around $\lambda=2.5 \mu \mathrm{m}$ (Z. Li et al.) [144]. (b) Conceptual diagram of the structure. An incident fundamental waveguide mode can couple into higher-order modes [144]. (c) Schematic of integrated metasurfaces consisting of nonlinear waveguide with patternning phase gradient metasurfaces on the top, which is used to achieve phase-matching-free second harmonic generation (Z. Li et al.) [145]. (d) Conceptual diagram of the phase-matching-free SHG. The optical power coupled from fundamental waveguide mode at the pump power to the fundamental waveguide mode at the SH frequency firstly, and then coupled to the higher-order waveguide modes at the SH frequency with the help of the metasurfaces [145].

\section{Incorporate graphene and other 2D materials into metasurfaces}

The optical properties of conventional nonlinear metasurface cannot be changed after fabrication. The conductivity of graphene allows the electric field to produce a subnanosecond response to field effect gating [146-150]. In order to overcome this shortcoming of conventional metasurfaces, the integration of graphene with plasmonic metasurfaces is recently designed to tune their optical properties by free carrier injection via field-effect gating. The generated strong optical fields and the enhanced optical absorption of graphene metasurfaces can serve as flexible platforms for ultra-planar nonlinear plasmonic devices [151-156].

\subsection{Enhanced nonlinear frequency generation}


Frequency conversion processes are usually implemented in nonlinear optics, providing opportunities for applications in photonics, chemistry, materials science and biosensing. In the discussion above, the method of harmonic generation in thin-film graphene is not strong enough due to the lack of strong light-matter interaction as observed in usually plasmonic materials. The metasurface can significantly improve the interaction between light and materials through the nano-scale gap between atoms, so as to improve the efficiency of the generation of second and third harmonics. Daria A et al. combine graphene plasmon mode with Fano resonance mode to design a novel graphene metasurface. As shown in Fig. 20 (a), the asymmetric mode of the metal nanoparticles and the graphene plasmon are coupled to each other, exhibiting strong mutual coupling and avoiding dispersion crossover. Fig. 20 (b) shows the SH field generated by the metasurface. The spectral position of the Fano resonance can be controlled by doping graphene, which promotes strong interactions between subradiation modes. Experiments confirm that this metasurface supports the cascaded Fano resonance caused by the sub-radiative plasmon mode of graphene and the local mode of the meta particles. The results show that combination of the sub-wavelength localization of plasmon can significantly enhance the nonlinear response and efficiency of the SHG [157]. F. Xiao et al. propose an electrically doped graphene metasurface in Fig. 20 (c), which regulates the Fermi energy of graphene and thus leads to the change of dielectric constant to actively control the SHG in the structure, as shown in Fig. 20 (d). Numerical simulations show that changes in the Fermi energy level of $30 \mathrm{meV}$ graphene can result in a 45 times SHG enhancement accompanied by a $220 \mathrm{~nm}$ resonance wavelength shift. According to perturbation theory, the real and imaginary parts of the dielectric constant of graphene dominate the resonance wavelength and SHG intensity respectively [158]. J. Chen et al. propose a novel nonlinear metasurface composed of single-layer $\mathrm{WS}_{2}$ and phase-controlled gold nanopore array as shown in Fig. 20 (e). The magnetic susceptibility of SHG is $10^{-1} \mathrm{~nm} / \mathrm{V}$ at $810 \mathrm{~nm}$, which is two or three orders of magnitude larger than the conventional plasmon metasurface. Fig. 20 (f) shows the intensity distribution of SHG along $\mathrm{x}$-axis, while the inset presents an optical image [159]. The metasurface composed of metal grating substrate and graphene sheet is proposed by J. Cao et al., as shown in Fig. 20 (g). The local electric field caused by the surface plasmon resonance of metal grating is employed to increase the nonlinear response of graphene, and the SPPs on the interface of the metal grating and graphene sheet are simultaneously excited. The simulation proves that the local electric field of the DFG beam increased the MIR generation efficiency by 3 orders of magnitude. MIR waves at various frequencies with different signal wavelengths and the corresponding 
graphene Fermi levels are plotted in Fig. 20 (h). By tuning the Fermi level of the graphene sheet, the tunable range of the MIR wave can reach 30 to $55 \mathrm{THz}$ [160].

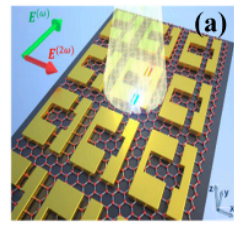

(b)

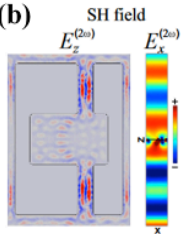

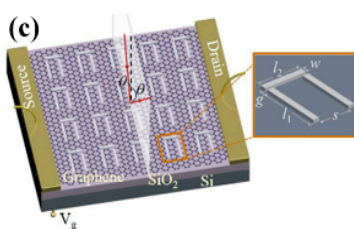

(d)





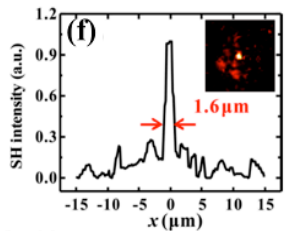

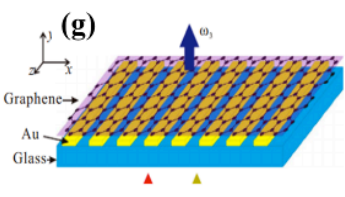

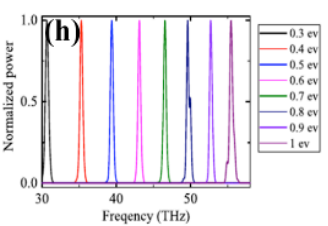

Fig. 20. Graphene metasurfaces to enhance SHG and THG. (a) Metasurface consisting of a graphene layer and an asymmetrical gold SRR lattice at the top (Daria A et al.). Reprinted with permission from [157]. Copyrights (2015) American Physical Society. (b) The SH field generated by the metasurface and the z-component of the electric field [157]. (c) Schematic diagram of dolmen structure based on single layer graphene (F. Xiao et al.). Reprinted with permission from [158]. Copyrights (2015) Optical Society of America. (d) SHG spectra of the dolmen structure as a function of graphene Fermi level [158]. (e) Proposed novel nonlinear metasurface composed of single-layer $\mathrm{WS}_{2}$ and phase-controlled gold nanopore array (J. Chen et al.). Reprinted with permission from [159]. Copyrights (2018) American Chemical Society. (f) The intensity distribution of SHG along x-axis. The inset presents an optical image [159]. (g) Schematic of the metasurface to enhance MIR generation. Two input beams at frequencies $\omega_{1}$ and $\omega_{2}$ generate the diff erence frequency beam $\omega_{3}$ (J. Cao et al.). Reprinted with permission from [160]. Copyrights (2017) Elsevier B.V. (h) MIR waves at various frequencies with different signal wavelengths and the corresponding graphene Fermi levels [160].

\subsection{Improvement of Raman signal}

Raman spectroscopy is known to play a key role in understanding the structure and properties of materials. In the past decades, an increasing attention has been drawn in studying the fundamental properties of graphene, and Raman spectroscopy has become a commonly used tool for understanding the behavior of electrons and phonons in graphene. In order to better understand the graphene information obtained from Raman spectroscopy, graphene-based metasurfaces are studied. The atomic level gap contributes greatly to the improvement of the interaction between light and matter and can significantly enhance the Raman signal. P. Huo et al. propose a hybrid metasurface that can effectively enhance the light-atomic graphene interaction. As presented in Fig. 21 (a), the hybrid metasurface is composed of randomly distributed silver NPs and a silver thin film with separation of dielectric spacers. The single- 
layer graphene sheet is tiled on the randomly distributed silver nanoparticles. Fig. 21 (b) shows Raman spectra of the graphene hybrid metasurface and graphene-silver nanoparticles. The SPR generated in the hybrid metasurface leads to a strong local electric field and broadband light absorption, which significantly enhances the light absorption of the atomic thin graphene layer and significantly enhances its original Raman signal. Compared with the metasurface of common graphene-silver nanoparticles [161], the mixed metasurface enhanced the Raman signal by more than 50 times [162]. X. Zhu at al. introduce a metasurface consisting of graphene coated to gold nanowire arrays in Fig. 21 (c), and nano-level gold cavity arrays significantly enhance light-matter interactions. The enhanced coupling between the plasmon mode of graphene and the nano-gap array results in significant frequency shifts of the plasmon resonance and a narrow and wide resonance peak in the whole visible and near-infrared regions. Fig. 21 (d) shows the Raman spectra of the metasurface and single graphene on $\mathrm{Si} / \mathrm{SiO}_{2}$ substrate. Experiments showed that the Raman response of graphene is enhanced up to 700 times. Numerical simulation and perturbation theory analysis further support the experimental observation. By placing Rhodamine 6G molecules on graphene, a strong enhancement of Rhodamine 6G Raman fingerprints is observed. These results pave the way for advanced surface-enhanced Raman scattering substrates [163]. F. Schedin et al. study surface-enhanced Raman scattering on metasurfaces on $\mathrm{SiO}_{2} / \mathrm{Si}$ substrates. As shown in Fig. 21 (e), the metasurface is composed of patterned graphene and the arrays of gold nanoparticles. Surface plasmons excited by metal nanoparticles can significantly increase Raman strength. Fig. 21 (f) shows the calculation of unpatterned single layer graphene on $\mathrm{SiO}_{2} / \mathrm{Si}$ and single layer graphene patterned with the Au nanodisks. The measurement results show that $\mathrm{G}$ and $2 \mathrm{D}$ are significantly enhanced at $633 \mathrm{~nm}$. It is also pointed out that Raman enhancement is directly proportional to the cross section of nano-particles and the fourth power Mie enhancement, and opposite to the separation of nano-particles and graphene. It means that thin nanodisks can achieve the highest Raman scattering of graphene [164]. B. Ghamsari et al. demonstrate the enhancement of graphene Raman signal based on stokes' plasmon resonance enhancement, as shown in Fig. 21 (g). Different from the laser pump field, here the enhancement originated from interactions with the Stokes field which can lead to different enhancement factors and spectral linewidths of $G$ and 2D peaks for the graphene characteristics, as plotted in Fig. 21 (h). The results show that the enhancement factor of Stokes field is up to 8 times as that of the initial pump field. The nano-antenna array provides a feasible means for the ultra-sensitive surface-enhanced Raman spectroscopy of graphene layers [165]. 

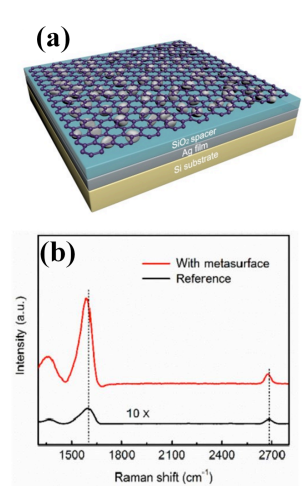

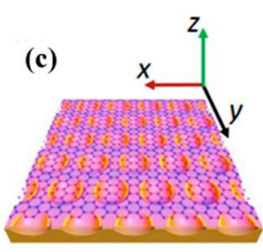

(e)
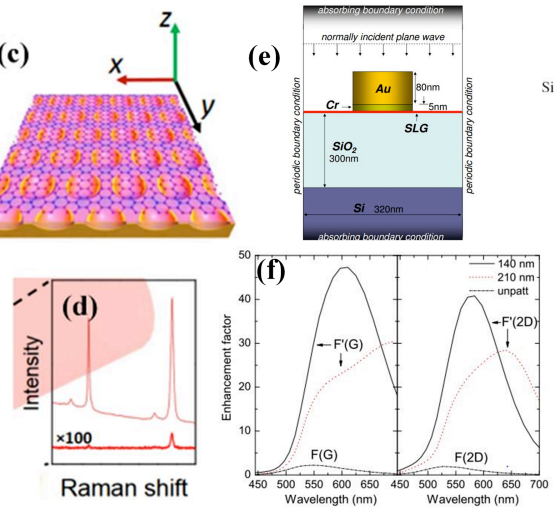
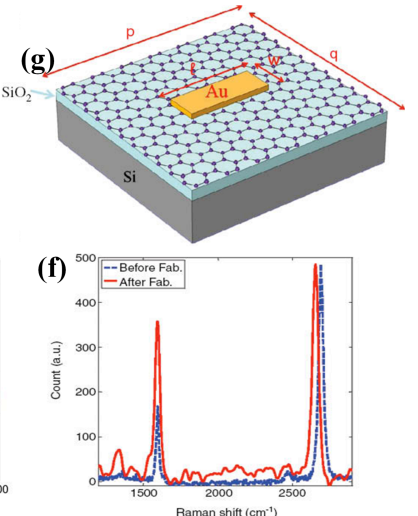

Fig. 21. Metasurface to enhance Raman spectroscopy of graphene. (a) The graphene-coated metal-dielectric-metal metasurface. The graphene is covered on the top of the metasurface. Silver nanoparticles are deposited on the $\mathrm{SiO}_{2}$ spacer (P. Huo et al) [162]. (b) Rama spectra of the graphene hybrid metasurface, compared with silver nanoparticles with the same distribution. [162]. (c) Schematic diagram of the nanovoid array covered by a monolayer graphene (X. Zhu at al.) [163]. (d) The Raman spectra of the metasurface and single graphene on $\mathrm{Si} / \mathrm{SiO}_{2}$ substrate [163]. (e) Unit cell of the metasurface consists of $\mathrm{Au} / \mathrm{Cr}$ nanodisks on $\mathrm{SLG} / \mathrm{SiO}_{2} / \mathrm{Si}$ (F. Schedin et al.) [164]. (f) The total enhancement factors for the G and $2 \mathrm{D}$ peaks. The dotted line is the corresponding interference enhancement factor [164]. (g) Schematic of the unit cell of the monopole nanoantenna arrays (B. Ghamsari et al) [165]. (h) Raman spectra on the graphene with and without the nanoantennas [165].

\subsection{Enhancement of FWM and phase conjugation}

In the case of degenerate FWM, two incident input pump and signal waves generate an idle wave, where the idle wave comes from the third-order nonlinear polarization caused by FWM in the nonlinear material. For unpatterned monolayer graphene-metal nanostructures, when surface plasmons are excited along the surface of graphene monolayer, the idle power outflow generated by FWM is much stronger than that of nanostructure without monolayer graphene. For a lower input frequency, the surface plasmon is difficult to be excited, but the nonlinear conductivity of graphene is stronger, resulting in stronger idle power value generated by FWM. To further improve the efficiency of FWM, B. Jin et al. replace graphene layers with patterned periodic graphene micron bands to form a graphene metasurface. Fig. 22(a) showed the structure of the graphene metasurface. The arrays of graphene microstrip are placed on a resonator composed of a dielectric layer and a gold film. The inset in Fig. 22(a) shows the electric field distribution for a $3 \mathrm{THz}$ resonance frequency, the frequencies of the pump and signal waves were approaching the resonance frequency of the metasurface. It can be seen that the electric field is significantly enhanced at the edge of the graphene band, and the FWM peak conversion efficiency can be improved by more than 5 orders of magnitude. Fig. 
22 (b) plots the power outflow of the idler wave generated by FWM. The idler power outflow decreases with increasing of the input waves incident angle, while the power reflected back into the surrounding space increases because of the low coupling between idler wave and metasurface. In a large range of incident angle, idler radiation can still maintain a large power value[166]. Phase conjugation is a special case of FWM, which can achieve negative refraction [167]. Due to its nano-scale properties, the phase matching of the FWM process is no longer related to the vertical component of the wave vector, but only depends on the transverse component along the graphene [168]. Therefore, by using FWM process, especially phase conjugation, negative refraction based on graphene films can be achieved. Two back-propagating pump waves are incident at an angle perpendicular to the graphene metasurface, as shown in Fig. 22 (c). If probe waves with the same frequency are incident at an oblique angle to the graphene metasurface, the transverse components of their wave vectors will be phase-matched, leading to phase conjugation. At this point, the pump and probe wave are overlaid because of the FWM process, resulting in a phase conjugation of the output and generating two negative refractive signals of the same frequency. Two output signals show negative reflection and refraction characteristics, respectively [169]. J. You et al. studied topologically protected FWM interactions in graphene metasurface, which consist of a periodic nanoholes array with hexagonal symmetry, as shown in the Fig. 22 (d). The metasurface exhibits a wide topological band gap at terahertz frequency after the timereversal symmetry of the static magnetic field is broken. In the case of the edge excitation, the light field propagates unidirectional along the surface edge of the graphene. In addition, this propagation process is robust to structural defects, which prevents it from backscattering. Fig. 22 (e) and (f) depict the near field distribution at pump, signal and idler frequencies. It can be seen that due to the nonlinear FWM interaction, signals are amplified during propagation and edge modes are generated at the free frequency. Moreover, the frequencies of all the interaction edge modes are located in the topology bandgap and protected by topology [170].

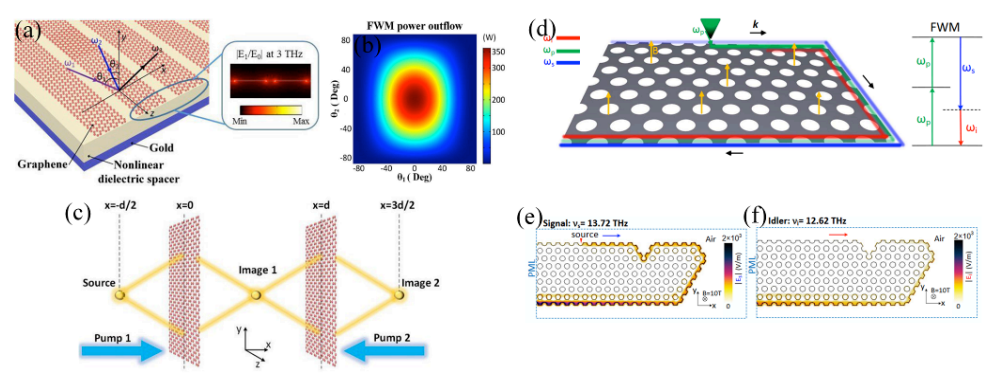

Fig. 22. (a) Schematic of the graphene metasurface to enhance FWM and Phase conjugation. Inset: The normalized electric field distribution (B. Jin et al.) [169]. (b) The power outflow 
generated by FWM as a function of the incident angle of the pump wave and the signal input wave [169]. (c) Combining two graphene monolayers with strong nonlinearity to realize subwavelength imaging based on phase conjugation. Reprinted with permission from [169]. Copyrights (2018) Society of Photo-Optical Instrumentation Engineers (SPIE). (d) Graphene metasurface with topologically protected FWM interactions, which consist of a periodic nanoholes array with hexagonal symmetry, and corresponding energy level diagram of the FWM process (J. You et al.) [170]. (e) The field profile at resonance frequency [170]. (f) The field profile at idle frequency [170].

\subsection{Efficient generation of high harmonics}

High harmonic generation (HHG) has been extensively studied in atomic gases in extreme ultraviolet and soft X-ray regions [171]. High harmonics in graphene can be induced by the coherent electron motion through strong light field. The typical high harmonic spectra of monolayer graphene excited by a linear polarized mid-infrared pulse is shown in Fig. 23(a), producing odd harmonics up to nine harmonics. N. Yoshikawa et al. investigated the effect of the ellipticity of laser on HHG. Fig. 23(b) shows the high harmonic spectra at various ellipticity. When the graphene is pumped with circularly polarized light (blue line), all harmonics disappear. The seventh and ninth harmonics excited by elliptic polarization pump (red line) are stronger than those by linear polarization pump (green line) [172]. In order to obtain compact and tunable light sources, metasurface -based light sources have been developed as passive compact radiation sources with free electrons [173]. However, most of the collective mode metasurface light sources are facing the challenges for material response at the X-ray frequency. Recently, light sources based on active graphene plasmon receive extensive attentions [174]. These free electron sources based on graphene plasmon have the potential to generate high photon energy without the need for high-intensity lasers. G. Rosolen et al. demonstrated a graphene metasurface that could significantly decrease the required electron energy and generate multiple harmonic X-ray radiation with near-field profiles. By utilizing the existence of high order spatial harmonics in metasurface-enhanced plasmons, multiple high order X-ray harmonics are generated by electron polaron scattering. The intensity and bandwidth of the emission can be controlled by a variety of parameters, including the design of the surface geometry, incident angle of the laser input, electron kinetic energy and pulse duration. The structure of proposed metasurface and the mechanism of metasurface mediated electromagnetic radiation is shown in Fig. 23 (c). Laser pulses excite local surface plasmons on the metasurface to modulate externally injected electrons, and electrons oscillating along the surface produce multiple harmonic x-rays. Specifically, the second order plasmon resonance of the graphene nano-ribbon (GNR) array is used to emit Xrays. Fig. 23 (d) compares the radiation intensity of the proposed graphene metasurface with 
that of unstructured graphene. It can be found that the graphene metasurface generates multiple harmonic X-rays and yields higher X-ray photon energies than pure graphene sheets. [175].

(a)
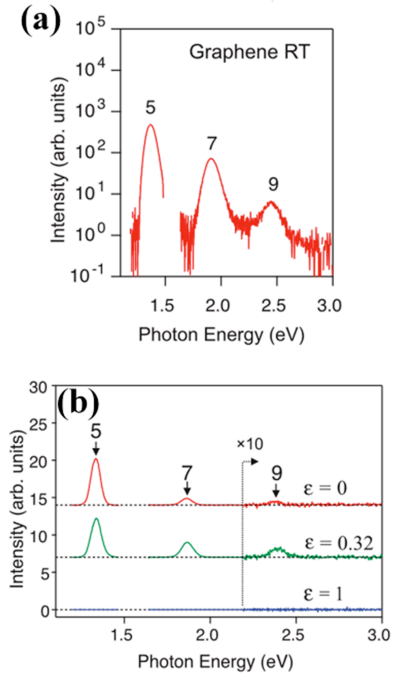

(c)


Fig. 23. (a) The typical high harmonic spectra of monolayer graphene excited by a linear polarized mid-infrared pulse (N. Yoshikawa et al.) [172]. (b) The high harmonic spectra at various ellipticity [172]. (c) Higher-harmonic X-rays from graphene metasurface based freeelectron light source (G. Rosolen et al.). Reprinted with permission from [175]. Copyrights (2018) Springer Nature. (d) The electron beam generates multiple harmonics dominated by the SH. Reprinted with permission from [175]. Copyrights (2018) Springer Nature.

\section{Summary and future outlook}

The nonlinear metasurfaces through tailoring subwavelength structures have led to the effective nonlinear optical process through the integration of patterned metasurface structures with nonlinear materials. In this review, we have discussed the recent progress of nonlinear metasurfaces. Our primary focus is the utilization of the local field enhancement induced by plasmonic excitation to improve the efficiency of nonlinear generations. We discussed the studies on nonlinear optical processes within designed nanostructures in planar metasurfaces, and also introduce the enhancement approaches for the detection of weak nonlinear optical processes by plasmonic nanosystems, especially by combining plasmonic metasurfaces with nonlinear materials. We also highlight the principles and applications of nonlinear graphene metasurfaces. The structures with pattern graphene exhibit more efficient optical processes and all-optical absorption than conventional plasmonic materials such as silver and gold nanofilms. In fact, planar technology greatly simplifies the manufacturing process compared to the $3 \mathrm{D}$ structure fabrication. Due to the greatly reduced manufacturing complexity, planar 
optical elements designed through metasurfaces are more suitable for large-scale applications. Several different lithography techniques for large-scale patterning of planar structures can be applied including nanoimprint lithography, deep UV lithography and soft lithography, etc.

With the further development of the functionality and performance of nonlinear metasurfaces, we foresee that nonlinear metasurfaces could have a significant impact on vortex light. The momentum of light possesses two freedoms, namely spin angular momentum (SAM) and orbital angular momentum (OAM). SAM is related to the circular polarization while OAM possesses a helical phase front, that can be measured by the topological charge and azimuthal angle, respectively. And the unbounded topological charge of OAM gives an infinite freedom for encoding optical information, makes it an important freedom for storing, controlling and transmitting information [176, 177]. In view of the phase and polarization control ability of metasurfaces, they can be designed to realize the control of OAM in the nonlinear process $[178,179]$. Different from linear devices, nonlinear metasurface is able to produce OAM beams at the harmonic wavelength for the incident beam, which is essential for extending the channels towards unconventional bands. Furthermore, the integration of 2D nonlinear materials into the metasurface can greatly enhance the harmonic conversion efficiency of the metasurface, providing the feasiblility by manipulating OAM of nonlinear optical signals.

In addition, the combination of quantum optics and metasurfaces has not been fully explored, but metasurfaces may have potential applications in quantum optics. The second and third order spontaneous parametric down-conversion, which can generate entangled photon pair and photon triplets have been attracted great attention. Basically, a device which is suitable for SHG or THG can also be employed for entangled quantum states generation. In this case, a light beam at higher frequency is required to excite the metasurface. It should be mentioned that direct generation of photon triplet denotes the Greenberger-Horne-Zeilinger (GHZ) state, which is particular important in fundamental quantum physics. The photon triplet generation in guiding wave devices such as optical fiber and plasmonic waveguide are already proposed [180,181], but the corresponding experimental results have not yet been validated. By taking the advantages of planar structure and continuous efficiency improvement, nonlinear metasurface could be the potential platform for quantum optics.

Modified silicene with a thickness of mono-layer silicon has become an emerging material. The SHG coefficient of 2D halogen and hydrogen-saturated silicene in the mid-infrared range is proven to be more than 10 times that of $\mathrm{KH}_{2} \mathrm{PO}_{4}$ [182]. MXene is an emerging 2D material composed of transition metal and carbides, nitrides or carbonitrides [183]. They have been reported to have a higher metal conductivity and their moldability, electromagnetic 
performance and stability than graphene. Moreover, a large number of different combinations of MXene can be designed for tuning their optical properties. The future research direction of metasurfaces combined with nonlinear 2D materials will focus on further exploring the nonlinear characteristics and applications of these new materials.

It is worth noting that the inherent losses in current plasmon systems is one of the important factors for the metasurfaces because they are directly linked to the nonlinear efficiency. Recent studies have reported that the field enhancement provided by the plasmon can provide compensation for nonlinear interactions [22]. In addition, recent research development on low-loss, tunable plasmonic materials such as transparent conducting oxide [184] and transition metal nitrides [185, 186] can be used as the novel substrates for low-loss metasurfaces. Thus, it enables the possibility to develop new metasurfaces with unparalleled characteristics, including larger working bandwidth with reduced loss. Another solution for this challenge is to design and fabricate the all-dielectric metasurfaces. By properly arranging the Mie-resonators with high nonlinear susceptibility to form metasurfaces, the loss will be significantly reduced while maintaining strong nonlinear response. In this case, one can design the metasurface by using material with low nonlinearity while combining the $2 \mathrm{D}$ material with high nonlinearity to enhance the conversion efficiency.

Another challenge for the metasurface devices is to enhance their conversion efficiencies to be comparable to those of nonlinear crystal. Thus, further improvement on the quality factors of the nonlinear devices are required e.g. by using the bound states in the continuum [187]. However, only enhancing the field strength can ultimately damage the structure and make the device more sensitive to the environmental noise. Another key point is to increase the capability of field collection. This requires specially-designed metasurfaces to satisfy field matching. Due to the high degrees of design freedom in metasurfaces for nonlinear applications, advanced design methods can be used for efficient optimization. Today, machine learning has become one of the dominant approaches in artificial intelligence to solve complex data driven problems. More importantly, this approach can be employed in metasurface as a powerful way to obtain the mapping between the topology and their associated functional features. With well-trained networks, inverse design of metasurface can be achieved without time-consuming electromagnetic calculation [188-190]. Currently, machine learning is mainly used for linear response prediction. With rapid development of artificial intelligence, on-demand design of nonlinear metasurface is foreseeable in the near future. 


\section{References}

[1] D. A. Zuev, S. V. Makarov, I. S. Mukhin, et al., "Fabrication of Hybrid Nanostructures via Nanoscale Laser-Induced Reshaping for Advanced Light Manipulation," Advanced Materials, 28(16), 3087-3093, 2016.

[2] S. Tsesses, G. Bartal, and I. Kaminer, "Light Generation via Quantum Interaction of Electrons with Periodic Nanostructures," Physical Review A, 95(1), 013832, 2017.

[3] C. Tan, C. Qin, and B. Sadtler, "Light-Directed Growth of Metal and Semiconductor Nanostructures," Journal of Materials Chemistry C, 5(23), 5628-5642, 2017.

[4] P. Vasa, and C. Lienau, "Strong Light-Matter Interaction in Quantum Emitter/Metal Hybrid Nanostructures," ACS Photonics, 5(1), 2-23, 2018.

[5] H. A. Elsayed, and S. A. El-Naggar, "Tuning the Flow of Light in Two-Dimensional Metallic Photonic Crystals Based on Faraday Effect AU - Aly, Arafa H," Journal of Modern Optics, 64(1), 74-80, 2017.

[6] K. O Brien, H. Suchowski, J. Rho, et al., "Predicting Nonlinear Properties of Metamaterials From the Linear Response," Nature Materials, 14(4), 379-383, 2015.

[7] J. B. Pendry, D. Schurig, and D. R. Smith, "Controlling Electromagnetic Fields," Science, 312(5781), 1780, 2006.

[8] U. Leonhardt, "Optical Conformal Mapping," Science, 312(5781), 1777, 2006.

[9] N. Yu, P. Genevet, F. Aieta, et al., "Flat Optics: Controlling Wavefronts With Optical Antenna Metasurfaces," IEEE Journal of Selected Topics in Quantum Electronics, 19(3), 4700423-4700423, 2013.

[10] N. Yu, P. Genevet, M. A. Kats, et al., "Light Propagation with Phase Discontinuities: Generalized Laws of Reflection and Refraction," Science (New York, N.Y.), 334(6054), 333$337,2011$.

[11] M. A. Kats, R. Blanchard, P. Genevet, et al., "Nanometre Optical Coatings Based on Strong Interference Effects in Highly Absorbing Media," Nature Materials, 12(1), 20-24, 2013.

[12] C. L. Holloway, E. F. Kuester, J. A. Gordon, et al., "An Overview of the Theory and Applications of Metasurfaces: The Two-Dimensional Equivalents of Metamaterials," IEEE Antennas and Propagation Magazine, 54(2), 10-35, 2012.

[13] A. V. Kildishev, A. Boltasseva, and V. M. Shalaev, "Planar Photonics with Metasurfaces," Science, 339(6125), 1232009, 2013.

[14] W. Cai, and V. Shalaev, "Optical Metamaterials: Fundamentals and Applications," Contemporary Physics, 53(3), 278-279, 2012. 
[15] D. S. Weile, "Electromagnetic Metamaterials: Physics and Engineering Explorations," IEEE Antennas and Propagation Magazine, 49(4), 137-139, 2007.

[16] R. A. Shelby, D. R. Smith, and S. Schultz, "Experimental Verification of a Negative Index of Refraction," Science, 292(5514), 77-79, 2001.

[17] L. Novotny, and N. van Hulst, "Antennas for Light," Nature Photonics, 5(83, 2011.

[18] P. Bharadwaj, B. Deutsch, and L. Novotny, "Optical Antennas," Advances in Optics and Photonics, 1(3), 438-483, 2009.

[19] D. M. Pozar, S. D. Targonski, and H. D. Syrigos, "Design of Millimeter Wave Microstrip Reflectarrays," IEEE Transactions on Antennas and Propagation, 45(2), 287-296, 1997.

[20] S. Larouche, and D. R. Smith, "Reconciliation of Generalized Refraction with Diffraction Theory," Optics Letters, 37(12), 2391-2393, 2012.

[21] X. Ni, N. K. Emani, A. V. Kildishev, et al., "Broadband Light Bending with Plasmonic Nanoantennas," Science, 335(6067), 427, 2012.

[22] M. Kauranen, and A. V. Zayats, "Nonlinear Plasmonics," Nature Photonics, 6(737, 2012.

[23] Y. R. Shen, "The Principles of Nonlinear Optics," Wiley-Interscience, 1984.

[24] A. V. Zayats, I. I. Smolyaninov, and A. A. Maradudin, "Nano-Optics of Surface Plasmon Polaritons," Physics Reports, 408(3), 131-314, 2005.

[25] M. I. Stockman, "Nanoplasmonics: Past, Present, and Glimpse into Future," Optics Express, 19(22), 22029-22106, 2011.

[26] S. A. Maier, "Plasmonics: Principles and Applications," Springer, 2007.

[27] E. Hendry, P. J. Hale, J. Moger, et al., "Coherent Nonlinear Optical Response of Graphene," Physical Review Letters, 105(9), 097401, 2010.

[28] S. Y. Hong, J. I. Dadap, N. Petrone, et al., "Optical Third-Harmonic Generation in Graphene," Physical Review X, 3(2), 021014, 2013.

[29] T. Gu, N. Petrone, J. F. McMillan, et al., "Regenerative Oscillation and Four-Wave Mixing in Graphene Optoelectronics," Nature Photonics, 6(554, 2012.

[30] H. Zhang, S. Virally, Q. Bao, et al., "Z-scan Measurement of the Nonlinear Refractive Index of Graphene," Optics Letters, 37(11), 1856-1858, 2012.

[31] A. V. Gorbach, A. Marini, and D. V. Skryabin, "Graphene-Clad Tapered Giber: Effective Nonlinearity and Propagation Losses," Optics Letters, 38(24), 5244-5247, 2013.

[32] C. Donnelly, and D. T. H. Tan, "Ultra-Large Nonlinear Parameter in Graphene-Silicon Waveguide Structures," Optics Express, 22(19), 22820-22830, 2014.

[33] Z. Fang, S. Thongrattanasiri, A. Schlather, et al., "Gated Tunability and Hybridization of Localized Plasmons in Nanostructured Graphene," ACS Nano, 7(3), 2388-2395, 2013. 
[34] Z. Fei, A. S. Rodin, G. O. Andreev, et al., "Gate-Tuning of Graphene Plasmons Revealed by Infrared Nano-Imaging," Nature, 487(82, 2012.

[35] F. H. L. Koppens, D. E. Chang, and F. J. García de Abajo, "Graphene Plasmonics: A Platform for Strong Light-Matter Interactions," Nano Letters, 11(8), 3370-3377, 2011.

[36] S. A. Mikhailov, and K. Ziegler, "New Electromagnetic Mode in Graphene," Physical Review Letters, 99(1), 016803, 2007.

[37] J. Chen, M. Badioli, P. Alonso-González, et al., "Optical Nano-Imaging of Gate-Tunable Graphene Plasmons," Nature, 487(77, 2012.

[38] M. Jablan, H. Buljan, and M. Soljačić, "Plasmonics in Graphene at Infrared Frequencies," Physical Review B, 80(24), 245435, 2009.

[39] A. Y. Nikitin, F. Guinea, F. J. Garcia-Vidal, et al., "Surface Plasmon Enhanced Absorption and Suppressed Transmission in Periodic Arrays of Graphene Ribbons," Physical Review B, 85(8), 081405, 2012.

[40] S. Y. Shin, N. D. Kim, J. G. Kim, et al., "Control of the $\pi$ Plasmon in a Single Layer Graphene by Charge doping," Applied Physics Letters, 99(8), 082110, 2011.

[41] Z. Fei, G. O. Andreev, W. Bao, et al., "Infrared Nanoscopy of Dirac Plasmons at the Graphene-SiO2 Interface," Nano Letters, 11(11), 4701-4705, 2011.

[42] Y. Liu, R. F. Willis, K. V. Emtsev, et al., "Plasmon Dispersion and Damping in Electrically Isolated Two-Dimensional Charge Sheets," Physical Review B, 78(20), 201403, 2008.

[43] C. Tegenkamp, H. Pfnür, T. Langer, et al., "Plasmon Electron-Hole Resonance in Epitaxial Graphene," Journal of physics. Condensed matter : an Institute of Physics journal, 23(1), 012001, 2011.

[44] Y. Liu, and R. F. Willis, "Plasmon-Phonon Strongly Coupled Mode in Epitaxial Graphene," Physical Review B, 81(8), 081406, 2010.

[45] J. H. Strait, P. Nene, W.-M. Chan, et al., "Confined Plasmons in Graphene Microstructures: Experiments and Theory," Physical Review B, 87(24), 241410, 2013.

[46] H. Yan, T. Low, W. Zhu, et al., "Damping Pathways of Mid-Infrared Plasmons in Graphene Nanostructures," Nature Photonics, 7(394, 2013.

[47] L. Ju, B. Geng, J. Horng, et al., "Graphene Plasmonics for Tunable Terahertz Metamaterials," Nature Nanotechnology, 6(630, 2011.

[48] Z. Fang, Y. Wang, A. E. Schlather, et al., "Active Tunable Absorption Enhancement with Graphene Nanodisk Arrays," Nano Letters, 14(1), 299-304, 2014.

[49] H. Yan, Z. Li, X. Li, et al., "Infrared Spectroscopy of Tunable Dirac Terahertz MagnetoPlasmons in Graphene," Nano Letters, 12(7), 3766-3771, 2012. 
[50] H. Yan, F. Xia, Z. Li, et al., "Plasmonics of Coupled Graphene Micro-Structures," New Journal of Physics, 14(12), 125001, 2012.

[51] H. Yan, X. Li, B. Chandra, et al., "Tunable infrared plasmonic devices using graphene/insulator stacks," Nature Nanotechnology, 7(330, 2012.

[52] V. W. Brar, M. S. Jang, M. Sherrott, et al., "Highly Confined Tunable Mid-Infrared Plasmonics in Graphene Nanoresonators," Nano Letters, 13(6), 2541-2547, 2013.

[53] S. Kujala, B. K. Canfield, M. Kauranen, et al., "Multipole Interference in the SecondHarmonic Optical Radiation from Gold Nanoparticles," Physical Review Letters, 98(16), 167403, 2007.

[54] R. W. Wood, "On a Remarkable Case of Uneven Distribution of Light in a Diffraction Grating Spectrum," Proceedings of the Physical Society of London, 18(1), 269-275, 1902.

[55] A. Otto, "Excitation of Nonradiative Surface Plasma Waves in Silver by the Method of Frustrated Total Reflection," Zeitschrift für Physik A Hadrons and nuclei, 216(4), 398-410, 1968.

[56] H. J. Simon, D. E. Mitchell, and J. G. Watson, "Optical Second-Harmonic Generation with Surface Plasmons in Silver Films," Physical Review Letters, 33(26), 1531-1534, 1974.

[57] George C. Schatz, and R. P. V. Duyne, "Electromagnetic Mechanism of Surfaceenhanced Spectroscopy," John Wiley \& Sons, Ltd, 2002.

[58] R. Jin, J. E. Jureller, H. Y. Kim, et al., "Correlating Second Harmonic Optical Responses of Single Ag Nanoparticles with Morphology," Journal of the American Chemical Society, 127(36), 12482-12483, 2005.

[59] M. Zavelani-Rossi, M. Celebrano, P. Biagioni, et al., "Near-Field Second-Harmonic Generation in Single Gold Nanoparticles," Applied Physics Letters, 92(9), 093119, 2008.

[60] M. Lippitz, M. A. van Dijk, and M. Orrit, "Third-Harmonic Generation from Single Gold Nanoparticles," Nano Letters, 5(4), 799-802, 2005.

[61] J. Butet, J. Duboisset, G. Bachelier, et al., "Optical Second Harmonic Generation of Single Metallic Nanoparticles Embedded in a Homogeneous Medium," Nano Letters, 10(5), 1717-1721, 2010.

[62] I. Russier-Antoine, E. Benichou, G. Bachelier, et al., "Multipolar Contributions of the Second Harmonic Generation from Silver and Gold Nanoparticles," The Journal of Physical Chemistry C, 111(26), 9044-9048, 2007.

[63] G. Bachelier, I. Russier-Antoine, E. Benichou, et al., "Multipolar Second-Harmonic Generation in Noble Metal Nanoparticles," Journal of the Optical Society of America B, 25(6), 955-960, 2008. 
[64] S. Roke, M. Bonn, and A. V. Petukhov, "Nonlinear optical scattering: The concept of effective susceptibility," Physical Review B, 70(11), 115106, 2004.

[65] M. Kauranen, "Freeing Nonlinear Optics from Phase Matching," Science, 342(6163), $1182,2013$.

[66] H. B. Jiang, L. Li, W. C. Wang, et al., "Reflected Second-Harmonic Generation at a Silver Surface," Physical Review B, 44(3), 1220-1224, 1991.

[67] J. Renger, R. Quidant, N. van Hulst, et al., "Surface-Enhanced Nonlinear Four-Wave Mixing," Physical Review Letters, 104(4), 046803, 2010.

[68] J. Butet, G. Bachelier, I. Russier-Antoine, et al., "Interference between Selected Dipoles and Octupoles in the Optical Second-Harmonic Generation from Spherical Gold Nanoparticles," Physical Review Letters, 105(7), 077401, 2010.

[69] S. D. Liu, E. S. P. Leong, G. C. Li, et al., "Polarization-Independent Multiple Fano Resonances in Plasmonic Nonamers for Multimode-Matching Enhanced Multiband SecondHarmonic Generation," ACS Nano, 10(1), 1442-1453, 2016.

[70] H. Aouani, M. Rahmani, M. Navarro Cía, et al., "Third-Harmonic-Upconversion Enhancement From a Single Semiconductor Nanoparticle Coupled to a Plasmonic Antenna," Nature Nanotechnology, 9(290, 2014.

[71] M. M. Glazov, "Second Harmonic Generation in Graphene," JETP Letters, 93(7), 366371, 2011.

[72] J. J. Dean, and H. M. van Driel, "Second Harmonic Generation from Graphene and Graphitic Films," Applied Physics Letters, 95(26), 261910, 2009.

[73] S. Wu, L. Mao, A. M. Jones, et al., "Quantum-Enhanced Tunable Second-Order Optical Nonlinearity in Bilayer Graphene," Nano Letters, 12(4), 2032-2036, 2012.

[74] J. L. Cheng, N. Vermeulen, and J. E. Sipe, "Third Order Optical Nonlinearity of Graphene," New Journal of Physics, 16(5), 053014, 2014.

[75] Q. H. Wang, K. Kalantar-Zadeh, A. Kis, et al., "Electronics and Optoelectronics of TwoDimensional Transition Metal Dichalcogenides," Nature Nanotechnology, 7(11), 699-712, 2012.

[76] S. Z. Butler, S. M. Hollen, L. Cao, et al., "Progress, Challenges, and Opportunities in Two-Dimensional Materials Beyond Graphene," ACS Nano, 7(4), 2898-2926, 2013.

[77] D. Jariwala, V. K. Sangwan, L. J. Lauhon, et al., "Emerging Device Applications for Semiconducting Two-Dimensional Transition Metal Dichalcogenides," ACS Nano, 8(2), 1102-1120, 2014. 
[78] J. Ribeiro-Soares, R. M. Almeida, E. B. Barros, et al., "Group theory analysis of phonons in two-dimensional transition metal dichalcogenides," Physical Review B, 90(11), 115438, 2014.

[79] S. Bergfeld, and W. Daum, "Second-Harmonic Generation in GaAs: Experiment versus Theoretical Predictions of $\mathrm{X}^{(2) x y z}, "$ Physical Review Letters, 90(3), 036801, 2003.

[80] S. Wang, H. Yu, H. Zhang, et al., "Broadband Few-Layer MoS2 Saturable Absorbers," Advanced Materials, 26(21), 3538-3544, 2014.

[81] Y. Takao, H. Asahina, and A. Morita, "Electronic Structure of Black Phosphorus in Tight Binding Approach," Journal of the Physical Society of Japan, 50(10), 3362-3369, 1981. [82] S. B. Lu, L. L. Miao, Z. N. Guo, et al., "Broadband nonlinear optical response in multilayer black phosphorus: an emerging infrared and mid-infrared optical material," Optics Express, 23(9), 11183-11194, 2015.

[83] J. Jackson, "Classical Electrodynamics," John Wiley \& Sons, Ltd, 2007.

[84] J. Butet, and O. J. F. Martin, "Evaluation of the Nonlinear Response of Plasmonic Metasurfaces: Miller's Rule, Nonlinear Effective Susceptibility Method, and Full-wave Computation," Journal of the Optical Society of America B, 33(2), A8-A15, 2016.

[85] H. Suchowski, K. O’Brien, Z. J. Wong, et al., "Phase Mismatch-Free Nonlinear Propagation in Optical Zero-Index Materials," Science, 342(6163), 1223, 2013.

[86] S. A. Mikhailov, "Theory of the Giant Plasmon-Enhanced Second-Harmonic Generation in Graphene and Semiconductor Two-Dimensional Electron Systems," Physical Review B, 84(4), 045432, 2011.

[87] G. Soavi, G. Wang, H. Rostami, et al., "Broadband, Electrically Tunable ThirdHarmonic Generation in Graphene," Nature Nanotechnology, 13(7), 583-588, 2018.

[88] M. A. Vincenti, D. de Ceglia, M. Grande, et al., "Third-Harmonic Generation In OneDimensional Photonic Crystal with Graphene-Based Defect," Physical Review B, 89(16), $165139,2014$.

[89] H. Zhou, T. Gu, J. F. McMillan, et al., "Enhanced Four-Wave Mixing in GrapheneSilicon Slow-Light Photonic Crystal Waveguides," Applied Physics Letters, 105(9), 091111, 2014.

[90] X. He, "Tunable Terahertz Graphene Metamaterials," Carbon, 82(229-237, 2015.

[91] H. Wang, J. Wu, J. Guo, et al., "Low-threshold optical bistability with multilayer graphene-covering Otto configuration," Journal of Physics D: Applied Physics, 49(25), 255306, 2016.

[92] V. A. Gorbach, "Graphene Plasmonic Waveguides for Mid-Infrared Supercontinuum Generation on a Chip," Photonics, 2(3), 2015. 
[93] J. D. Cox, A. Marini, and F. J. G. de Abajo, "Plasmon-assisted high-harmonic generation in graphene," Nature communications, 8(14380, 2017.

[94] T. Guo, and C. Argyropoulos, "Broadband Polarizers based on Graphene Metasurfaces," Optics Letters, 41(23), 5592-5595, 2016.

[95] P. A. Huidobro, M. Kraft, S. A. Maier, et al., "Graphene as a Tunable Anisotropic or Isotropic Plasmonic Metasurface," ACS Nano, 10(5), 5499-5506, 2016.

[96] Z. Li, K. Yao, F. Xia, et al., "Graphene Plasmonic Metasurfaces to Steer Infrared Light," Scientific Reports, 5(12423, 2015.

[97] M. M. Jadidi, J. C. König-Otto, S. Winnerl, et al., "Nonlinear Terahertz Absorption of Graphene Plasmons," Nano Letters, 16(4), 2734-2738, 2016.

[98] H. Nasari, M. S. Abrishamian, and P. Berini, "Nonlinear Optics of Surface Plasmon Polaritons in Subwavelength Graphene Ribbon Resonators," Optics Express, 24(1), 708-723, 2016.

[99] F. Ling, G. Yao, and J. Yao, "Active Tunable Plasmonically Induced Polarization Conversion in the THz Regime," Scientific Reports, 6(34994, 2016.

[100] K. L. Seyler, J. R. Schaibley, P. Gong, et al., "Electrical control of second-harmonic generation in a WSe2 monolayer transistor," Nature Nanotechnology, 10(407, 2015.

[101] X. Lin, Y. Liu, K. Wang, et al., "Two-Dimensional Pyramid-like WS2 Layered Structures for Highly Efficient Edge Second-Harmonic Generation," ACS Nano, 12(1), 689696, 2018.

[102] D. Li, C. Wei, J. Song, et al., "Anisotropic Enhancement of Second Harmonic Generation in Monolayer and Bilayer MoS2 by Integrating with TiO2 Nanowires," 2019.

[103] M. Klein, B. H. Badada, R. Binder, et al., "2D Semiconductor Nonlinear Plasmonic Modulators," Nature communications, 10(1), 3264, 2019.

[104] G. Aivazian, H. Yu, S. Wu, et al., "Many-body Effects in Nonlinear Optical Responses of 2D Layered Semiconductors," 2D Materials, 4(2), 025024, 2017.

[105] M. Silveirinha, and N. Engheta, "Tunneling of Electromagnetic Energy through Subwavelength Channels and Bends using epsilon-Near-Zero Materials," Physical Review Letters, 97(15), 157403, 2006.

[106] A. Ciattoni, C. Rizza, and E. Palange, "Extreme Nonlinear Electrodynamics in Metamaterials with very Small Linear Dielectric Permittivity," Physical Review A, 81(4), 043839, 2010.

[107] M. A. Vincenti, D. de Ceglia, A. Ciattoni, et al., "Singularity-Driven Second- and Third-Harmonic Generation at Epsilon-Near-Zero Crossing Points," Physical Review A, 84(6), 063826, 2011. 
[108] A. Ciattoni, and E. Spinozzi, "Efficient Second-harmonic Generation in Micrometerthick Slabs with Indefinite Permittivity," Physical Review A, 85(4), 043806, 2012.

[109] V. M. Agranovich, and V. E. Kravtsov, "Notes on Crystal Optics of Superlattices," Solid State Communications, 55(1), 85-90, 1985.

[110] B. M. Wells, W. Guo, and V. A. Podolskiy, "Homogenization of Nanowire-based Composites with Anisotropic Unit-cell and Layered Substructure," MRS Communications, 6(1), 23-29, 2016.

[111] A. Y. B. Brian Wells, Giuseppe Marino, Mazhar E. Nasir, Anatoly V. Zayats, and Viktor A. Podolskiy, "Structural second-order nonlinearity in plasmonic metamaterials," Optical Materials Express, 5(12), 1502-1507, 2018.

[112] A. D. Neira, N. Olivier, M. E. Nasir, et al., "Eliminating Material Constraints for Nonlinearity with Plasmonic Metamaterials," Nature communications, 6(1), 7757, 2015.

[113] A. Capretti, Y. Wang, N. Engheta, et al., "Enhanced Third-harmonic Generation in Sicompatible Epsilon-near-zero Indium Tin Oxide Nanolayers," Optics Letters, 40(7), 1500$1503,2015$.

[114] N. Kinsey, C. DeVault, J. Kim, et al., "Epsilon-near-zero Al-doped ZnO for ultrafast switching at telecom wavelengths," Optica, 2(7), 616-622, 2015.

[115] M. Z. Alam, I. De Leon, and R. W. Boyd, "Large optical nonlinearity of indium tin oxide in its epsilon-near-zero region," Science, 352(6287), 795, 2016.

[116] R. W. Boyd, "Nonlinear Optics," Elsevier, 1992.

[117] A. R. Davoyan, A. M. Mahmoud, and N. Engheta, "Optical Isolation with Epsilon-nearzero Metamaterials," Optics Express, 21(3), 3279-3286, 2013.

[118] N. Meinzer, W. L. Barnes, and I. R. Hooper, "Plasmonic Meta-atoms and Metasurfaces," Nature Photonics, 8(12), 889-898, 2014.

[119] G. Vampa, B. G. Ghamsari, S. Siadat Mousavi, et al., "Plasmon-enhanced Highharmonic Generation from Silicon," Nature Physics, 13(659, 2017.

[120] B. K. Canfield, S. Kujala, K. Jefimovs, et al., "Linear and Nonlinear Optical Responses Influenced by Broken Symmetry in an Array of Gold Nanoparticles," Optics Express, 12(22), 5418-5423, 2004.

[121] M. Tymchenko, J. S. Gomez-Diaz, J. Lee, et al., "Gradient Nonlinear PancharatnamBerry Metasurfaces," Physical Review Letters, 115(20), 207403, 2015.

[122] S. C. Jiang, X. Xiong, Y. S. Hu, et al., "High-Efficiency Generation of Circularly Polarized Light via Symmetry-Induced Anomalous Reflection," Physical Review B, 91(12), $125421,2015$. 
[123] Z. Li, W. Liu, Z. Li, et al., "Tripling the Capacity of Optical Vortices by Nonlinear Metasurface," Laser \& Photonics Reviews, 12(11), 1800164, 2018.

[124] W. Li, and J. Valentine, "Metamaterial Perfect Absorber Based Hot Electron Photodetection," Nano Letters, 14(6), 3510-3514, 2014.

[125] P. Fan, Z. Yu, S. Fan, et al., "Optical Fano Resonance of an Individual Semiconductor Nanostructure," Nature Materials, 13(471, 2014.

[126] Z. Liu, H. Shao, G. Liu, et al., " $\lambda 3 / 20000$ Plasmonic Nanocavities with Multispectral Ultra-Narrowband Absorption for High-Quality Sensing," Applied Physics Letters, 104(8), $081116,2014$.

[127] P. P. Vabishchevich, S. Liu, M. B. Sinclair, et al., "Enhanced Second-Harmonic Generation Using Broken Symmetry III-V Semiconductor Fano Metasurfaces," ACS Photonics, 5(5), 1685-1690, 2018.

[128] M. R. Shcherbakov, D. N. Neshev, B. Hopkins, et al., "Enhanced Third-Harmonic Generation in Silicon Nanoparticles Driven by Magnetic Response," Nano Letters, 14(11), 6488-6492, 2014.

[129] Y. Yang, W. Wang, A. Boulesbaa, et al., "Nonlinear Fano-Resonant Dielectric Metasurfaces," Nano Letters, 15(11), 7388-7393, 2015.

[130] S. Liu, M. B. Sinclair, S. Saravi, et al., "Resonantly Enhanced Second-Harmonic Generation Using III-V Semiconductor All-Dielectric Metasurfaces," Nano Letters, 16(9), 5426-5432, 2016.

[131] J. A. Bossard, and D. H. Werner, "Metamaterials with Custom Emissivity Polarization in the Near-infrared," Optics Express, 21(3), 3872-3884, 2013.

[132] M. W. Klein, C. Enkrich, M. Wegener, et al., "Second-Harmonic Generation from Magnetic Metamaterials," Science, 313(5786), 502, 2006.

[133] C. Wu, N. Arju, G. Kelp, et al., "Spectrally Selective Chiral Silicon Metasurfaces based on Infrared Fano Resonances," Nature communications, 5(3892, 2014.

[134] S. Campione, A. Benz, J. F. Klem, et al., "Electrodynamic Modeling of Strong Coupling between a Metasurface and Intersubband Transitions in Quantum Wells," Physical Review B, 89(16), 165133, 2014.

[135] O. Wolf, S. Campione, Y. Yang, et al., "Multipolar Second Harmonic Generation in a Symmetric Nonlinear Metamaterial," Scientific Reports, 7(1), 8101, 2017.

[136] A. Benz, S. Campione, S. Liu, et al., "Strong coupling in the Sub-wavelength Limit using Metamaterial Nanocavities," Nature communications, 4(1), 2882, 2013.

[137] O. Wolf, S. Campione, A. Benz, et al., "Phased-array Sources based on Nonlinear Metamaterial Nanocavities," Nature communications, 6(1), 7667, 2015. 
[138] Y. Todorov, A. M. Andrews, R. Colombelli, et al., "Ultrastrong Light-Matter Coupling Regime with Polariton Dots," Physical Review Letters, 105(19), 196402, 2010.

[139] W. Fan, S. Zhang, K. J. Malloy, et al., "Second Harmonic Generation From Patterned GaAs inside a Subwavelength Metallic Hole Array," Optics Express, 14(21), 9570-9575, 2006.

[140] N. Feth, S. Linden, M. W. Klein, et al., "Second-Harmonic Generation from Complementary Split-Ring Resonators," Optics Letters, 33(17), 1975-1977, 2008.

[141] F. B. P. Niesler, N. Feth, S. Linden, et al., "Second-Harmonic Generation from SplitRing Resonators on a GaAs Substrate," Optics Letters, 34(13), 1997-1999, 2009.

[142] J. Lee, M. Tymchenko, C. Argyropoulos, et al., "Giant Nonlinear Response from Plasmonic Metasurfaces Coupled to Intersubband Transitions," Nature, 511(65, 2014.

[143] R. Sarma, D. de Ceglia, N. Nookala, et al., "Broadband and Efficient Second-Harmonic Generation from a Hybrid Dielectric Metasurface/Semiconductor Quantum-Well Structure," ACS Photonics, 6(6), 1458-1465, 2019.

[144] Z. Li, M. Kim, C. Wang, et al., "Controlling Propagation and Coupling of Waveguide Modes Using Phase-Gradient Metasurfaces," Nature Nanotechnology, 12(675, 2017.

[145] C. Wang, Z. Li, M. Kim, et al., "Metasurface-Assisted Phase-Matching-Free Second Harmonic Generation in Lithium Niobate Waveguides," Nature communications, 8(1), 2098, 2017.

[146] M. Liu, X. Yin, and X. Zhang, "Double-Layer Graphene Optical Modulator," Nano Letters, 12(3), 1482-1485, 2012.

[147] A. Vakil, and N. Engheta, "Fourier Optics on Graphene," Physical Review B, 85(7), 075434, 2012.

[148] M. Liu, X. Yin, E. Ulin-Avila, et al., "A graphene-based Broadband Optical Modulator," Nature, 474(64, 2011.

[149] S. H. Lee, M. Choi, T. Kim, et al., "Switching Terahertz Waves with Gate-Controlled Active Graphene Metamaterials," Nature Materials, 11(936, 2012.

[150] A. Vakil, and N. Engheta, "Transformation Optics Using Graphene," Science, 332(6035), 1291, 2011.

[151] Y. Yao, M. A. Kats, P. Genevet, et al., "Broad Electrical Tuning of Graphene-Loaded Plasmonic Antennas," Nano Letters, 13(3), 1257-1264, 2013.

[152] N. K. Emani, T. Chung, A. V. Kildishev, et al., "Electrical Modulation of Fano Resonance in Plasmonic Nanostructures Using Graphene," Nano Letters, 14(1), 78-82, 2014. 
[153] N. Dabidian, I. Kholmanov, A. B. Khanikaev, et al., "Electrical Switching of Infrared Light Using Graphene Integration with Plasmonic Fano Resonant Metasurfaces," ACS Photonics, 2(2), 216-227, 2015.

[154] C. Wu, A. B. Khanikaev, R. Adato, et al., "Fano-Resonant Asymmetric Metamaterials for Ultrasensitive Spectroscopy and Identification of Molecular Monolayers," Nature Materials, 11(69, 2011.

[155] S. H. Mousavi, I. Kholmanov, K. B. Alici, et al., "Inductive Tuning of Fano-Resonant Metasurfaces Using Plasmonic Response of Graphene in the Mid-Infrared," Nano Letters, 13(3), 1111-1117, 2013.

[156] Z. Li, and N. Yu, "Modulation of Mid-Infrared Light Using Graphene-Metal Plasmonic Antennas," Applied Physics Letters, 102(13), 131108, 2013.

[157] D. A. Smirnova, A. E. Miroshnichenko, Y. S. Kivshar, et al., "Tunable Nonlinear Graphene Metasurfaces," Physical Review B, 92(16), 161406, 2015.

[158] F. Xiao, W. Zhu, W. Shang, et al., "Electrical Control of Second Harmonic Generation in a Graphene-Based Plasmonic Fano Structure," Optics Express, 23(3), 3236-3244, 2015.

[159] J. Chen, K. Wang, H. Long, et al., "Tungsten Disulfide-Gold Nanohole Hybrid Metasurfaces for Nonlinear Metalenses in the Visible Region," Nano Letters, 18(2), 1344$1350,2018$.

[160] J. Cao, Y. Kong, S. Gao, et al., "Plasmon Resonance Enhanced Mid-Infrared Generation by Graphene on Gold Gratings through Difference Frequency Mixing," Optics Communications, 406(183-187, 2018.

[161] Y. Du, Y. Zhao, Y. Qu, et al., "Enhanced Light-Matter Interaction of Graphene-Gold Nanoparticle Hybrid Films for High-Performance SERS Detection," Journal of Materials Chemistry C, 2(23), 4683-4691, 2014.

[162] P. Huo, Y. Liang, S. Zhang, et al., "Hybrid Metasurface for Broadband Enhancing Optical Absorption and Raman Spectroscopy of Graphene," Optical Materials Express, 7(10), 3591-3597, 2017.

[163] X. Zhu, L. Shi, M. S. Schmidt, et al., "Enhanced Light-Matter Interactions in Graphene-Covered Gold Nanovoid Arrays," Nano Letters, 13(10), 4690-4696, 2013.

[164] F. Schedin, E. Lidorikis, A. Lombardo, et al., "Surface-Enhanced Raman Spectroscopy of Graphene," ACS Nano, 4(10), 5617-5626, 2010.

[165] B. G. Ghamsari, A. Olivieri, F. Variola, et al., "Enhanced Raman Scattering in Graphene by Plasmonic Resonant Stokes Emission," Nanophotonics, 3(6), 0014, 2014. 
[166] B. Jin, and C. Argyropoulos, "Nonlinear Graphene Metasurfaces with Advanced Electromagnetic Functionalities," Plasmonics: Design, Materials, Fabrication, Characterization, and Applications, 107221R(2018.

[167] S. Maslovski, and S. Tretyakov, "Phase Conjugation and Perfect Lensing," Journal of Applied Physics, 94(7), 4241-4243, 2003.

[168] S. M. Rao, A. Lyons, T. Roger, et al., "Geometries for the Coherent Control of FourWave Mixing in Fraphene Multilayers," Scientific Reports, 5(15399, 2015.

[169] C. A. Boyuan Jin, Christos Argyropoulos, "Nonlinear graphene metasurfaces with advanced electromagnetic functionalities," Proc. SPIE, 10722(2018.

[170] Z. L. J. You, N. Panoiu, "Four-wave Mixing of Topological Edge Plasmons in a Graphene Metasurface," Nonlinear Optics, 2019.

[171] P. B. Corkum, and F. Krausz, "Attosecond science," Nature Physics, 3(6), 381-387, 2007.

[172] N. Yoshikawa, T. Tamaya, and K. Tanaka, "High-Harmonic Generation In Graphene Enhanced by Elliptically Polarized Light Excitation," Science, 356(6339), 736, 2017.

[173] G. Adamo, J. Y. Ou, J. K. So, et al., "Electron-Beam-Driven Collective-Mode Metamaterial Light Source," Physical Review Letters, 109(21), 217401, 2012.

[174] L. J. Wong, I. Kaminer, O. Ilic, et al., "Towards Graphene Plasmon-Based FreeElectron Infrared to X-ray Sources," Nature Photonics, 10(46, 2015.

[175] G. Rosolen, L. J. Wong, N. Rivera, et al., "Metasurface-Based Multi-Harmonic FreeElectron Light Source," Light: Science \& Applications, 7(1), 64, 2018.

[176] X. Wang, X. Cai, Z. Su, et al., "Quantum Teleportation of Multiple Degrees of Freedom of a Single Photon," Nature, 518(7540), 516-519, 2015.

[177] J. Wang, J.-Y. Yang, I. M. Fazal, et al., "Terabit Free-Space Data Transmission Employing Orbital Angular Momentum Multiplexing," Nature Photonics, 6(7), 488-496, 2012.

[178] G. Li, L. Wu, K. F. Li, et al., "Nonlinear Metasurface for Simultaneous Control of Spin and Orbital Angular Momentum in Second Harmonic Generation," Nano Letters, 17(12), 7974-7979, 2017.

[179] A. Dasgupta, X. Yang, and J. Gao, "Nonlinear Conversion of Orbital Angular Momentum in Tungsten Disulfide Monolayer," Journal of Optics, 21(12), 125404, 2019.

[180] A. Cavanna, F. Just, X. Jiang, et al., "Hybrid Photonic-Crystal Fiber for Single-Mode Phase Matched Generation of Third Harmonic and Photon Triplets," Optica, 3(9), 952-955, 2016 . 
[181] T. Huang, J. Pan, Z. Cheng, et al., "Photon-plasmon Coupling for Fundamental-mode Phase-matched Third Harmonic and Triplet Photon Generation," Journal of Lightwave Technology, 36(18), 3892-3897, 2018.

[182] G. Yang, and K. Wu, "Absorption and Mid-IR SHG in Two-Dimensional Halogen and Hydrogen Saturated Silicene Series," The Journal of Physical Chemistry C, 121(48), 2713927146, 2017.

[183] M. Naguib, M. Kurtoglu, V. Presser, et al., "Two-Dimensional Nanocrystals Produced by Exfoliation of Ti3AlC2," Advanced Materials, 23(37), 4248-4253, 2011.

[184] P. R. West, S. Ishii, G. V. Naik, et al., "Searching for Better Plasmonic Materials," Laser \& Photonics Reviews, 4(6), 795-808, 2010.

[185] G. V. Naik, J. L. Schroeder, X. Ni, et al., "Titanium Nitride as a Plasmonic Material for Visible and Near-Infrared Wavelengths," Optical Materials Express, 2(4), 478-489, 2012.

[186] G. V. Naik, J. Kim, and A. Boltasseva, "Oxides and Nitrides as Alternative Plasmonic Materials in the Optical Range [Invited]," Optical Materials Express, 1(6), 1090-1099, 2011.

[187] L. Carletti, K. Koshelev, C. De Angelis, et al., "Giant Nonlinear Response at the Nanoscale Driven by Bound States in the Continuum," Physical Review Letters, 121(3), 033903, 2018.

[188] Z. Liu, D. Zhu, S. P. Rodrigues, et al., "Generative Model for the Inverse Design of Metasurfaces," Nano Letters, 18(10), 6570-6576, 2018.

[189] J. Jiang, D. Sell, S. Hoyer, et al., "Free-Form Diffractive Metagrating Design Based on Generative Adversarial Networks," ACS Nano, 13(8), 8872-8878, 2019.

[190] J. Jiang, and J. A. Fan, "Global Optimization of Dielectric Metasurfaces Using a Physics-Driven Neural Network," Nano Letters, 19(8), 5366-5372, 2019. 